\title{
Signatures of bosonic squark decays in non-minimally flavour-violating supersymmetry
}

\author{
Matthias Bruhnke, ${ }^{a}$ Björn Herrmann ${ }^{b, a}$ and Werner Porod ${ }^{a, c}$ \\ ${ }^{a}$ Institut für Theoretische Physik und Astrophysik, Universität Würzburg, \\ D-97074 Würzburg, Germany \\ ${ }^{b}$ Theory group, Deutsches Elektronen-Synchrotron (DESY), \\ Notkestraße 85, D-22603 Hamburg, Germany \\ ${ }^{c}$ AHEP Group, Institut de Física Corpuscular - C.S.I.C., Universitat de València, \\ E-46071 València, Spain \\ E-mail: mbruhnke@physik.uni-wuerzburg.de, bjoern.herrmann@desy.de, \\ porod@physik.uni-wuerzburg.de
}

ABSTRACT: We investigate couplings of squarks to gauge and Higgs-bosons within the framework of non-minimal flavour violation in the Minimal Supersymmetric Standard Model. Introducing non-diagonal elements in the mass matrices of squarks, we first study their impact on the self-energies and physical mass eigenvalues of squarks. We then present an extensive analysis of bosonic squark decays for variations of the flavour-violating parameters around the two benchmark scenarios SPS1a' and SPS1b. Signatures, that would be characteristic for a non-minimal flavour structure in the squark sector, can be found in wide regions of the parameter space.

KEYWords: Supersymmetry Phenomenology

ARXIV EPRINT: 1007.2100 


\section{Contents}

1 Introduction 1

2 Quark flavour violation in the MSSM 2

3 Impact of quark flavour violation on squark masses 4

4 Quark flavour violating decays of squarks into bosons 9

$\begin{array}{lll}4.1 & \text { Signatures for the variation of one NMFV-parameter } & 10\end{array}$

$\begin{array}{lll}4.2 & \text { Signatures for the variation of two NMFV-parameters } & 15\end{array}$

$\begin{array}{llr}5 & \text { Conclusion } & 20\end{array}$

$\begin{array}{lr}\text { A Couplings } & 22\end{array}$

$\begin{array}{ll}\text { A.1 Squark-squark-vector couplings } & 22\end{array}$

$\begin{array}{ll}\text { A.2 Squark-squark-Higgs couplings } & 23\end{array}$

$\begin{array}{ll}\text { B One-loop mass matrices } & 25\end{array}$

\section{Introduction}

With the start of the Large Hadron Collider (LHC) first measurements at the TeV-scale will be realized in near future. Although the Standard Model (SM) of particle physics has given most accurate predictions for a wide range of phenomena, there are strong hints that it is not the ultimate theory, but should rather be thought of as a low-energy limit of a more fundamental framework. Among the numerous candidates for the latter, the Minimal Supersymmetric Standard Model (MSSM) is probably the best-studied extension of the Standard Model. Postulating a superpartner with opposite statistics for each SM particle, supersymmetry (SUSY) cures the hierarchy problem by stabilising the Higgs mass, can lead to gauge-coupling unification, and includes natural candidates for the dark matter observed in our Universe. Although it is clear that supersymmetry must be broken at the electroweak scale, there is no theoretical consensus about the exact breaking mechanism. One therefore introduces so-called soft SUSY breaking parameters which do not introduce quadratic divergences. Thus, the stability of the ratio of the electroweak scale over a GUT scale or the Planck scale is maintained. It will be the task of LHC and other future colliders like the ILC to measure these parameters as precisely as possible [1] which then will give a clue on how SUSY is broken.

One of the open questions related to the breaking mechanism concerns the flavour structure of the MSSM. The hypothesis of minimal flavour violation (MFV) [2] assumes that it is the same as in the Standard Model, where all quark flavour-violating interactions 
are parametrised through the CKM-matrix. However, in principle new sources of flavour violation can appear within supersymmetric models, especially if they are embedded in larger frameworks such as grand unified theories [3-5]. This so-called non-minimal flavour violation (NMFV) allows for non-diagonal, i.e. flavour-violating, entries in the mass matrices at the weak scale, that cannot be related to the CKM-matrix any more. These entries are conveniently taken as additional free parameters and can imply a different phenomenology as compared to the case of MFV. For a more detailed review on flavour violation in the MSSM see, e.g., ref. [6].

In the last years, various publications have focused on the phenomenology of nonminimal flavour violation in the squark sector of the MSSM. In refs. [7] and [8], loop-induced effects on the decays of top-quarks or Higgs-bosons have been investigated, respectively. Various flavour-changing neutral current processes related to the top-quark have been discussed in refs. $[9,10]$. The production of squarks and gauginos at hadron colliders has been studied in refs. [11, 12], and in refs. [13-16] possible signatures of gluino and squark decays have been discussed.

The aim of the present paper is to provide a complementary study in the context of bosonic decays of squarks. Therefore, we investigate the impact of new sources of flavour violation on the couplings of the squarks to gauge and Higgs-bosons. Such flavour-violating couplings can induce new contributions to the squark mass matrices, either at the tree-level or at the one-loop level or both. These contributions influence directly the mass spectrum and the flavour decomposition of the different squark mass eigenstates. Concerning the decays into vector bosons or Higgs-bosons, NMFV in the squark sector can allow for interesting signatures already at tree-level, that would not be possible within the framework of MFV. If squarks are discovered at the LHC and the relevant signals can be revealed in the decay chains, they would allow to exclude the hypothesis of MFV in the MSSM.

This paper is organised as follows. In section 2, we review the parametrisation of flavour violation in the squark sector of the MSSM and the most relevant experimental constraints. We then discuss in section 3 the impact of NMFV on the squark mass spectrum at the one-loop level and the induced flavour-mixing terms. We finally study experimental signatures related to flavour-violating squark decays into final states containing gauge and Higgs-bosons in section 4. Conclusions are given in section 5 .

\section{Quark flavour violation in the MSSM}

In supersymmetric models with the most general form of flavour mixing, the $6 \times 6$ squark mass matrices have the form

$$
\mathcal{M}_{\tilde{q}}^{2}=\left(\begin{array}{cc}
\mathcal{M}_{\tilde{q}, L L}^{2} & \mathcal{M}_{\tilde{q}, L R}^{2} \\
\mathcal{M}_{\tilde{q}, R L}^{2} & \mathcal{M}_{\tilde{q}, R R}^{2}
\end{array}\right),
$$

for $\tilde{q}=\tilde{u}, \tilde{d}$, respectively. The $3 \times 3$ diagonal blocks of the mass matrices are given by

$$
\begin{array}{rlrl}
\mathcal{M}_{\tilde{d}, L L}^{2} & =\hat{m}_{\tilde{q}}^{2}+D_{\tilde{d}, L L} \mathbf{1}+\hat{m}_{d}^{2}, & & \mathcal{M}_{\tilde{u}, L L}^{2}=V_{\mathrm{CKM}} \hat{m}_{\tilde{q}}^{2} V_{\mathrm{CKM}}^{\dagger}+D_{\tilde{d}, L L} \mathbf{1}+\hat{m}_{u}^{2}, \\
\mathcal{M}_{\tilde{d}, R R}^{2}=\hat{m}_{\tilde{d}}^{2}+D_{\tilde{d}, R R} \mathbf{1}+\hat{m}_{d}^{2}, & \mathcal{M}_{\tilde{u}, R R}^{2}=\hat{m}_{\tilde{u}}^{2}+D_{\tilde{u}, R R} \mathbf{1}+\hat{m}_{u}^{2},
\end{array}
$$


where $\hat{m}_{\tilde{q}, \tilde{d}, \tilde{u}}$ are the soft-breaking mass parameters of the squarks in the super-CKM basis and $\hat{m}_{u, d}$ denote the diagonal mass matrices of up- and down-type quarks. The D-terms are $D_{\tilde{q}, L L}=\cos 2 \beta m_{Z}^{2}\left(T_{3}^{q}-e_{q} \sin ^{2} \theta_{W}\right)$ and $D_{\tilde{q}, R R}=e_{q} \sin ^{2} \theta_{W} \cos 2 \beta m_{Z}^{2}$. Here, $T_{3}^{q}$ and $e_{q}$ denote the isospin and electric charge of the (s)quarks, and $\theta_{W}$ is the weak mixing angle. Due to the SU(2) symmetry, the left-left blocks are related through the CKM-matrix $V_{\mathrm{CKM}}$. The off-diagonal blocks of eq. (2.1) are given by

$$
\begin{aligned}
& \mathcal{M}_{\tilde{d}, R L}^{2}=\mathcal{M}_{\tilde{d}, L R}^{2 \dagger}=\frac{v_{d}}{\sqrt{2}} \hat{T}_{D}-\mu^{*} \hat{m}_{d} \tan \beta, \\
& \mathcal{M}_{\tilde{u}, R L}^{2}=\mathcal{M}_{\tilde{u}, L R}^{2 \dagger}=\frac{v_{u}}{\sqrt{2}} \hat{T}_{U}-\mu^{*} \hat{m}_{u} \cot \beta,
\end{aligned}
$$

where $\hat{T}_{D, U}$ are the trilinear soft-breaking parameters in the super-CKM basis. The parameters related to the Higgs sector are the ratio of the vacuum expectation values of the two Higgs doublets, $\tan \beta=v_{u} / v_{d}$, and the higgsino mass parameter $\mu$.

In order to diagonalize the mass matrices of eq. (2.1), two $6 \times 6$ rotation matrices $\mathcal{R}_{\tilde{u}}$ and $\mathcal{R}_{\tilde{d}}$ are needed, defined such that

$$
\operatorname{diag}\left(m_{\tilde{q}_{1}}^{2}, \ldots, m_{\tilde{q}_{6}}^{2}\right)=\mathcal{R}_{\tilde{q}} \mathcal{M}_{\tilde{q}}^{2} \mathcal{R}_{\tilde{q}}^{\dagger} \quad \text { and } \quad m_{\tilde{q}_{1}}<\cdots<m_{\tilde{q}_{6}} .
$$

For the sake of a dimensionless and scenario-independent description, non-minimal flavour violation (NMFV) in the squark sector is conveniently parametrised by the parameters $\delta_{i j}^{I J}$ defined through

$$
\begin{aligned}
\left(\hat{m}_{\tilde{q}}^{2}\right)_{i j} & =\delta_{i j}^{L L} \frac{1}{12}\left[2 \operatorname{Tr}\left\{\hat{m}_{\tilde{q}}^{2}\right\}+\operatorname{Tr}\left\{\hat{m}_{\tilde{u}}^{2}\right\}+\operatorname{Tr}\left\{\hat{m}_{\tilde{d}}^{2}\right\}\right] \\
\left(\hat{m}_{\tilde{d}}^{2}\right)_{i j} & =\delta_{i j}^{d, R R} \frac{1}{6}\left[\operatorname{Tr}\left\{\hat{m}_{\tilde{q}}^{2}\right\}+\operatorname{Tr}\left\{\hat{m}_{\tilde{d}}^{2}\right\}\right], \\
\left(\hat{m}_{\tilde{u}}^{2}\right)_{i j} & =\delta_{i j}^{u, R R} \frac{1}{6}\left[\operatorname{Tr}\left\{\hat{m}_{\tilde{q}}^{2}\right\}+\operatorname{Tr}\left\{\hat{m}_{\tilde{u}}^{2}\right\}\right], \\
\frac{v_{d}}{\sqrt{2}}\left(\hat{T}_{D}\right)_{i j} & =\delta_{i j}^{d, R L} \frac{1}{6}\left[\operatorname{Tr}\left\{\hat{m}_{\tilde{q}}^{2}\right\}+\operatorname{Tr}\left\{\hat{m}_{\tilde{d}}^{2}\right\}\right], \\
\frac{v_{d}}{\sqrt{2}}\left(\hat{T}_{D}^{*}\right)_{i j} & =\delta_{i j}^{d, L R} \frac{1}{6}\left[\operatorname{Tr}\left\{\hat{m}_{\tilde{q}}^{2}\right\}+\operatorname{Tr}\left\{\hat{m}_{\tilde{d}}^{2}\right\}\right], \\
\frac{v_{u}}{\sqrt{2}}\left(\hat{T}_{U}\right)_{i j} & =\delta_{i j}^{u, R L} \frac{1}{6}\left[\operatorname{Tr}\left\{\hat{m}_{\tilde{q}}^{2}\right\}+\operatorname{Tr}\left\{\hat{m}_{\tilde{u}}^{2}\right\}\right], \\
\frac{v_{u}}{\sqrt{2}}\left(\hat{T}_{U}^{*}\right)_{i j} & =\delta_{i j}^{u, L R} \frac{1}{6}\left[\operatorname{Tr}\left\{\hat{m}_{\tilde{q}}^{2}\right\}+\operatorname{Tr}\left\{\hat{m}_{\tilde{u}}^{2}\right\}\right],
\end{aligned}
$$

for $i, j=1,2,3$ (and $i \neq j$ ). Note that with this definition one can account for potential large left-right mixing effects as the traces correspond roughly to the sum of the squark masses squared at tree level.

Many experimental measurements impose constraints on the parameter space of the MSSM. In the context of flavour transitions, the most relevant constraints come from precision measurements of mixing and decays of $K$ - and $B$-mesons, where the squarks enter at the same loop-level as the standard model contributions. In particular, very stringent constraints are imposed on generation mixing involving first generation squarks [20-22]. We take them implicitly into account by considering only mixing between second and third generation squarks, which is least constrained. In particular, this means that we consider seven independent NMFV parameters,

$$
\begin{array}{ll}
\delta^{L L} \equiv \delta_{23}^{L L}, \quad \delta_{u}^{R R} \equiv \delta_{23}^{u, R R}, & \delta_{d}^{R R} \equiv \delta_{23}^{d, R R}, \quad \delta_{u}^{R L} \equiv \delta_{23}^{u, R L}=\left(\delta_{32}^{u, L R}\right)^{*} \\
\delta_{u}^{L R} \equiv \delta_{23}^{u, L R}=\left(\delta_{32}^{u, R L}\right)^{*}, & \delta_{d}^{R L} \equiv \delta_{23}^{d, R L}=\left(\delta_{32}^{d, L R}\right)^{*}, \quad \delta_{d}^{L R} \equiv \delta_{23}^{d, L R}=\left(\delta_{32}^{d, R L}\right)^{*},
\end{array}
$$




\begin{tabular}{|c|c|c|}
\hline Observable & Exp. value and error & Theor. uncertainty \\
\hline $10^{4} \times \mathrm{BR}(b \rightarrow s \gamma)$ & $3.52 \pm 0.23 \pm 0.09[26]$ & ${ }_{-0.49}^{+0.37}[27,28]$ \\
$\Delta M_{B_{s}}\left[\mathrm{ps}^{-1}\right]$ & $17.77 \pm 0.10 \pm 0.07[29]$ & $\pm 3.88[30]$ \\
$10^{6} \times \mathrm{BR}\left(\bar{B}_{s} \rightarrow X_{s} l^{+} l^{-}\right)$ & $1.60 \pm 0.50[31]$ & $\pm 0.11[31]$ \\
$10^{8} \times \mathrm{BR}\left(B_{s} \rightarrow \mu^{+} \mu^{-}\right)$ & $\leq 3.6[26]$ & \\
$m_{h^{0}}[\mathrm{GeV}]$ & $\geq 114.4[29]$ & $\pm 3.0[32]$ \\
\hline
\end{tabular}

Table 1. Constraints on flavour violation in the squark sector, current experimental limits and theoretical error estimates.

where we omit for simplicity the generation indices. The so-defined parameters are assumed to be real, the influence of possible complex phases being beyond the scope of this work.

We then explicitly impose the constraints given in table 1 on the flavour mixing between second and third generation squarks. The experimental upper and lower limits on $\mathrm{BR}\left(B_{s} \rightarrow\right.$ $\mu^{+} \mu^{-}$) and $m_{h^{0}}$ are given at the $95 \%$ confidence level, while the error intervals for the other observables are given at the $68 \%(1 \sigma)$ confidence level.

The calculation of the physical mass spectrum and the rotation matrices as well as the observables shown in table 1 is done using SPheno 3.0 [23]. ${ }^{1}$ Furthermore, in order to perform scans over the parameter space in an efficient way on a computer cluster, we make use of the Mathematica package MapCore described in ref. [24].

The scenarios discussed in the following are in agreement with the the current experimental limits given above at $95 \%$ confidence level for wide ranges of the parameters of eq. (2.6). Here, we also take into account the available theoretical error estimates given in table 1. A detailed study of the allowed ranges in the parameter space is, however, beyond the scope of this paper. For selected parameter configurations, we shall in section 4 indicate the allowed ranges of the NMFV-parameters $\delta_{q}^{I J}$. Detailed studies of constraints on the MSSM parameter space due to precision measurements and low-energy observables can also be found in refs. [9-12, 14-19].

\section{Impact of quark flavour violation on squark masses}

The following study is based on the two reference scenarios SPS1a' [33] and SPS1b [34] in the framework of minimal supergravity (mSUGRA) to fix the flavour diagonal parameters. The corresponding input parameters at the grand unification scale are the universal scalar mass $m_{0}=70(200) \mathrm{GeV}$, gaugino mass $m_{1 / 2}=250(400) \mathrm{GeV}$, and trilinear coupling $A_{0}=-300(0) \mathrm{GeV}$ for SPS1a' (SPS1b). At the GUT scale we take $T_{f}=A_{0} Y_{f}$ where $Y_{f}$ is the corresponding Yukawa coupling. In contrast $\tan \beta=10$ (30) is given at the scale $m_{Z}$. The higgsino mass parameter $\mu$ is positive for both scenarios. For the input values of the standard model parameters we refer the reader to ref. [29]. The mass of the top quark has been set to $m_{t}^{\text {pole }}=172.9 \mathrm{GeV}$. The soft-breaking terms and the mass spectrum at

${ }^{1}$ An updated version including flavour effects can be obtained at
http://www.physik.uni-wuerzburg.de/ porod/SPheno.html.




\begin{tabular}{|c|c|c|c|}
\hline SPS1a' & \multicolumn{2}{|c|}{ Flavour content } & Mass \\
\hline$\tilde{d}_{6}$ & $96 \% \tilde{d}_{L}$ & $4 \% \tilde{s}_{L}$ & 570 \\
\hline$\tilde{d}_{5}$ & $96 \% \tilde{s}_{L}$ & $4 \% \tilde{d}_{L}$ & 570 \\
\hline$\tilde{d}_{4}$ & $100 \% \tilde{d}_{R}$ & & 547 \\
\hline$\tilde{d}_{3}$ & $100 \% \tilde{s}_{R}$ & & 547 \\
\hline$\tilde{d}_{2}$ & $91 \% \tilde{b}_{R}$ & $9 \% \tilde{b}_{L}$ & 546 \\
\hline$\tilde{d}_{1}$ & $91 \% \tilde{b}_{L}$ & $9 \% \tilde{b}_{R}$ & 506 \\
\hline$\tilde{u}_{6}$ & $68 \% \tilde{t}_{L}$ & $32 \% \tilde{t}_{R}$ & 586 \\
\hline$\tilde{u}_{5}$ & $99 \% \tilde{u}_{L}$ & $1 \% \tilde{c}_{L}$ & 565 \\
\hline$\tilde{u}_{4}$ & $99 \% \tilde{c}_{L}$ & $1 \% \tilde{u}_{L}$ & 565 \\
\hline$\tilde{u}_{3}$ & $100 \% \tilde{u}_{R}$ & & 547 \\
\hline$\tilde{u}_{2}$ & $100 \% \tilde{c}_{R}$ & & 547 \\
\hline$\tilde{u}_{1}$ & $68 \% \tilde{t}_{R}$ & $32 \% \tilde{t}_{L}$ & 367 \\
\hline
\end{tabular}

\begin{tabular}{|c|c|c|c|}
\hline SPS1b & \multicolumn{2}{|c|}{ Flavour content } & Mass \\
\hline$\tilde{d}_{6}$ & $98 \% \tilde{d}_{L}$ & $2 \% \tilde{s}_{L}$ & 888 \\
\hline$\tilde{d}_{5}$ & $98 \% \tilde{s}_{L}$ & $2 \% \tilde{d}_{L}$ & 888 \\
\hline$\tilde{d}_{4}$ & $100 \% \tilde{d}_{R}$ & & 852 \\
\hline$\tilde{d}_{3}$ & $100 \% \tilde{s}_{R}$ & & 852 \\
\hline$\tilde{d}_{2}$ & $73 \% \tilde{b}_{R}$ & $27 \% \tilde{b}_{L}$ & 831 \\
\hline$\tilde{d}_{1}$ & $73 \% \tilde{b}_{L}$ & $27 \% \tilde{b}_{R}$ & 783 \\
\hline$\tilde{u}_{6}$ & $99 \% \tilde{c}_{L}$ & $1 \% \tilde{u}_{L}$ & 884 \\
\hline$\tilde{u}_{5}$ & $99 \% \tilde{u}_{L}$ & $1 \% \tilde{c}_{L}$ & 884 \\
\hline$\tilde{u}_{4}$ & $100 \% \tilde{u}_{R}$ & & 855 \\
\hline$\tilde{u}_{3}$ & $100 \% \tilde{c}_{R}$ & & 855 \\
\hline$\tilde{u}_{2}$ & $76 \% \tilde{t}_{L}$ & $24 \% \tilde{t}_{R}$ & 846 \\
\hline$\tilde{u}_{1}$ & $76 \% \tilde{t}_{R}$ & $24 \% \tilde{t}_{L}$ & 667 \\
\hline
\end{tabular}

Table 2. Flavour content and masses of up- and down-type squarks for the reference scenario SPS1a' (left) and SPS1b (right) assuming MFV. The masses are given in GeV.

the SUSY scale $Q=1 \mathrm{TeV}$ are obtained through two-loop renormalization group (RGE) running [25]. The calculation of the physical masses is done at the one-loop level, including leading two-loop contributions to the Higgs masses. Here we have included flavour effects in the renormalization group (RGE) running as well as in the calculation of the masses and mixing matrices.

In table 2 we show the resulting physical masses and the corresponding flavour decomposition of the up- and down-type squarks in the case of minimal flavour violation (MFV) for the two reference scenarios. For both points the left-right mixing is most important for third generation squarks. Due to the larger value of $\tan \beta$ in the case of SPS1b the sbottom mixing is larger w.r.t. SPS1a'. The small mixings involving the first and second generation are CKM-induced both in the RGE running of the parameters as well as in corresponding entries of the one-loop mass matrices. Since here the CKM-matrix is the only source of flavour mixing, this is relevant for left-handed squarks only.

In the following, we focus mainly on the scenario SPS1a' for a detailed discussion of variations around the MFV-case, and discuss additional effects induced due to the larger value of $\tan \beta$ using SPS1b. We add at the electroweak scale additional NMFV-parameters to the MFV-parameters obtained in the RGE running and study their effects on masses and mixing. As a first example, let us study the dependence on $\delta_{d}^{R R}$. This parameter induces a direct $\tilde{s}_{R}-\tilde{b}_{R}$ mixing and affects at tree-level the down-sector only and at the one-loop level a tiny mixing is induced in the up-sector as well. In figure 1 , we show the mass eigenvalues and the flavour decomposition of selected down-type squarks. For $\delta_{d}^{R R}=0, \tilde{d}_{3}$ is a pure $\tilde{s}_{R}$ state and almost mass-degenerate with $\tilde{d}_{2}$, as can be seen from table 2 . Due to the small mass difference, a strong $\tilde{s}_{R}-\tilde{b}_{R}$ mixing is observed already for small values of $\delta_{d}^{R R}$. This leads to several sharp level-crossings between the mass eigenstates when the flavour 

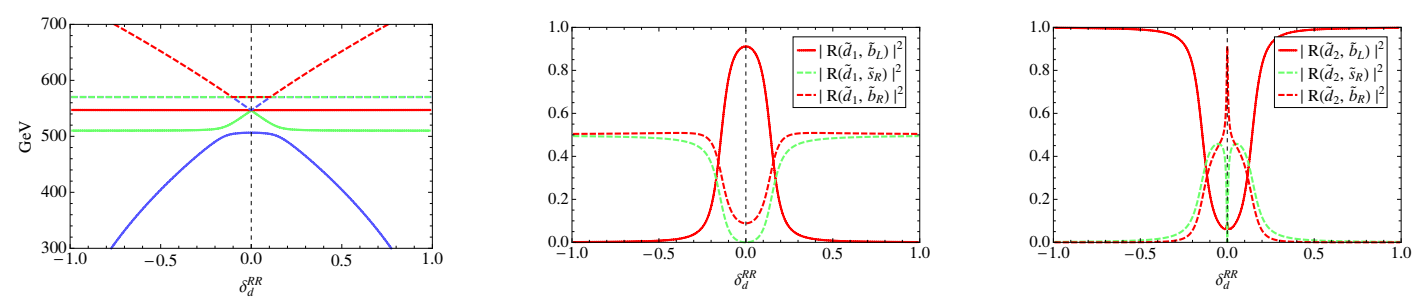

Figure 1. Dependence of the masses (left) and the flavour decomposition (centre and right) of selected down-type squarks on the NMFV-parameter $\delta_{d}^{R R}$ based on the benchmark scenario SPS1a'. The point $\delta_{d}^{R R}=0$ corresponds to the MFV-scenario of table 2 .
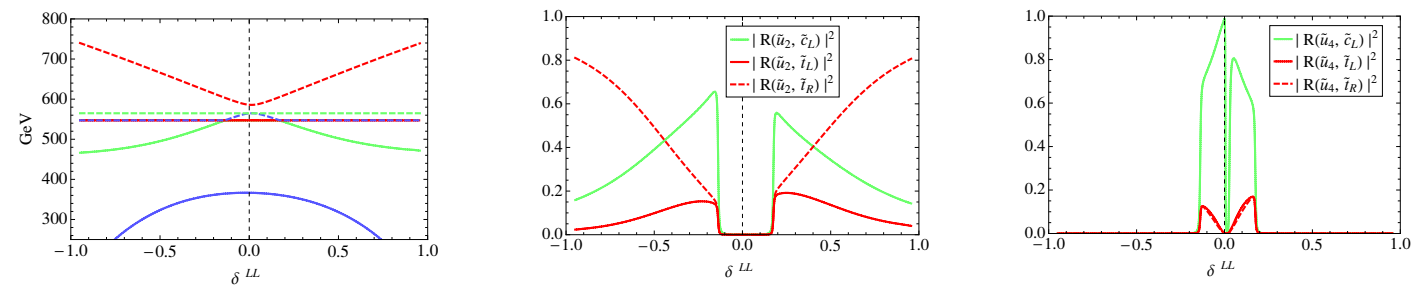

Figure 2. Dependence of the masses (left) and the flavour decomposition (centre and right) of selected up-type squarks on the NMFV-parameter $\delta^{L L}$ based on the benchmark scenario SPS1a'. The point $\delta^{L L}=0$ corresponds to the MFV-scenario of table 2 .

contents of the corresponding squarks are exchanged. A rather smooth crossing occurs around $\delta_{d}^{R R} \approx 0.2$ when the $\tilde{b}_{L}$ content of $\tilde{d}_{1}$ is taken over by $\tilde{d}_{2}$.

A similar situation is present in the sector of up-type squarks for a variation of the parameter $\delta_{u}^{R R}$. The only difference is the larger mass gap already in the MFV case (see table 2). As a consequence, the exchange of flavour contents between the u-squarks is less pronounced.

Due to $\mathrm{SU}(2)$ invariance a non-zero parameter $\delta^{L L}$ induces both a $\tilde{c}_{L^{-}} \tilde{t}_{L}$ and a $\tilde{s}_{L^{-}}$ $\tilde{b}_{L}$ mixing. In figure we show the squark mass eigenvalues and flavour decompositions of selected up-type squarks as a function of $\delta^{L L}$ while all other NMFV-parameters of eq. (2.6) are kept to zero. Again, level-crossings and flavour exchanges are observed already for moderate values of $\delta^{L L}$. Note that here the behaviour of the up-type squarks is not symmetric w.r.t. the MFV-case, which is a consequence of the CKM-relation between the two left-left blocks of the mass matrices. In consequence, small $\tilde{u}_{L}-\tilde{c}_{L}$ and $\tilde{u}_{L}-\tilde{t}_{L}$ mixing are induced in addition to $\tilde{c}_{L}-\tilde{t}_{L}$. The fact that this asymmetry shows in the up-sector is due to the definition of the super-CKM basis, where the down-type squarks are diagonal.

Let us now turn to flavour-violating entries in the off-diagonal blocks of the squark mass matrices. Figure 3 shows the dependence of up-type squark mass eigenvalues and flavour contents as a function of $\delta_{d}^{R L}$. Since this parameter affects not only the mass matrix but primarily the squark-squark-Higgs coupling, we observe significant differences to the previous cases. First, instead of an increasing mass splitting, the two lightest downtype squark masses decrease both with increasing $\delta_{d}^{R L}$, implying that the trace of the mass matrix is not invariant under the influence of the NMFV-parameter.

Indeed, the parameter $\delta_{d}^{R L}$ induces squark self-energies stemming from the Higgs-loops 

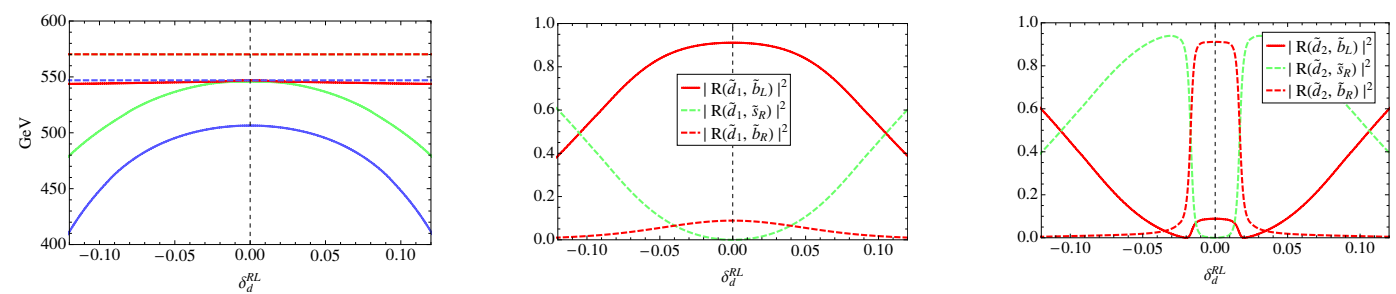

Figure 3. Dependence of masses (left) and flavour decompositions (centre and right) of selected down-type squarks on the NMFV-parameter $\delta_{d}^{R L}$ based on the benchmark scenario SPS1a'. The point $\delta_{d}^{R L}=0$ corresponds to the MFV-scenario of table 2 .

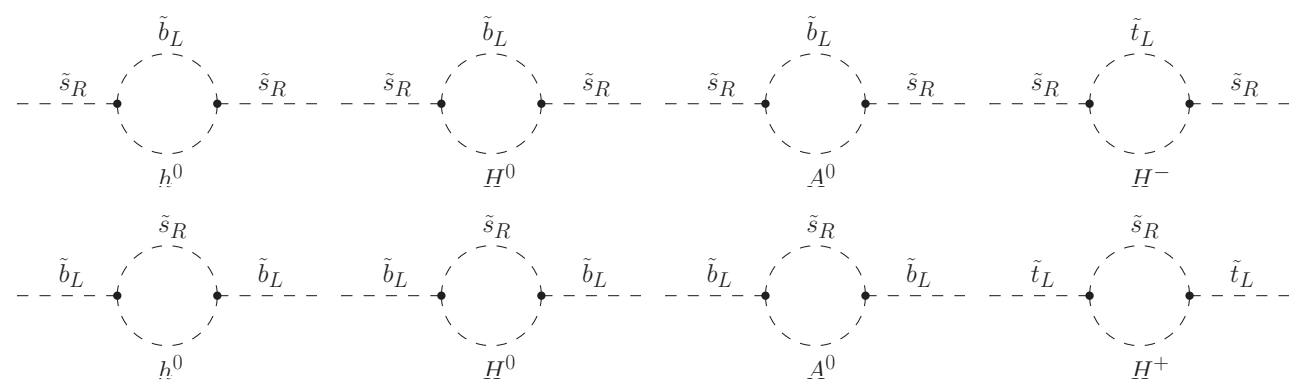

Figure 4. One-loop contributions from Higgs-bosons to the self-energies of right-handed strange and left-handed bottom and top squarks.

shown in figure 4 , that are quadratic in the squark-squark-Higgs coupling $\left(\hat{T}_{D}\right)_{23}$. In the limit where these contributions dominate we get from eqs. (B.2) and (B.3)

$$
\Delta m^{2} \simeq-\left|\left(\hat{T}_{D}\right)_{23}\right|^{2} B_{0}\left(p^{2}, m_{\tilde{q}}^{2}, m_{H}^{2}\right),
$$

where we denote schematically $m_{\tilde{q}}$ and $m_{H}$ the masses of the squarks and Higgs bosons in the loop, respectively. The main contributions are due to due $H^{0}, A^{0}$ and $H^{+}$where those including a charged Higgs are about twice as large as the loops containing $H^{0}$ or $A^{0}$. Assuming approximately equal masses for $H^{0}, A^{0}$, and $H^{ \pm}$(see table 2), the mass parameter of $\tilde{s}_{R}$ receives a two times larger corrections than the mass parameter of $\tilde{s}_{L}$. Note, that independent of the sign of $\left(\hat{T}_{D}\right)_{23}$ this contributions will reduce the trace of the down squark mass matrix compared to the MFV case leading to the observed reduction of the two lightest states.

The resulting decrease of the diagonal elements overcompensates the increase of the off-diagonal ones. In consequence, the strong mixing of the two lightest down-type squark states is reversed for $\delta_{d}^{R L} \rightarrow \pm 1$. This "unmixing" effect is observed, e.g., in figure 3 for $\left|\delta_{d}^{R L}\right| \gtrsim 0.12$. Due to the loop with a charged Higgs-boson (see figure 4), the parameter $\delta_{d}^{R L}$ also affects the sector of up-type squarks. In particular, the mass parameter of $\tilde{t}_{L}$ receives a correction of similar size than its isospin partner $\tilde{b}_{L}$. For this reason, also the two stop-states unmix for large $\left|\delta_{d}^{R L}\right|$.

The most interesting NMFV-parameters are those inducing off-diagonal entries in the trilinear couplings of up-type squarks. In the mSUGRA scenarios under consideration here, the diagonal entry $\left(\hat{T}_{U}\right)_{33}$ is relatively large due to large top Yukawa coupling. This does 


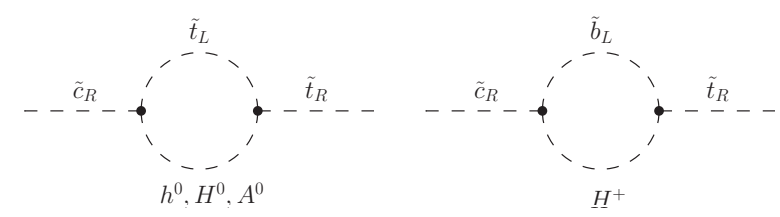

Figure 5. One-loop contributions to $\tilde{c}_{R}-\tilde{t}_{R}$ mixing that are linear in the parameter $\delta_{u}^{R L}$.
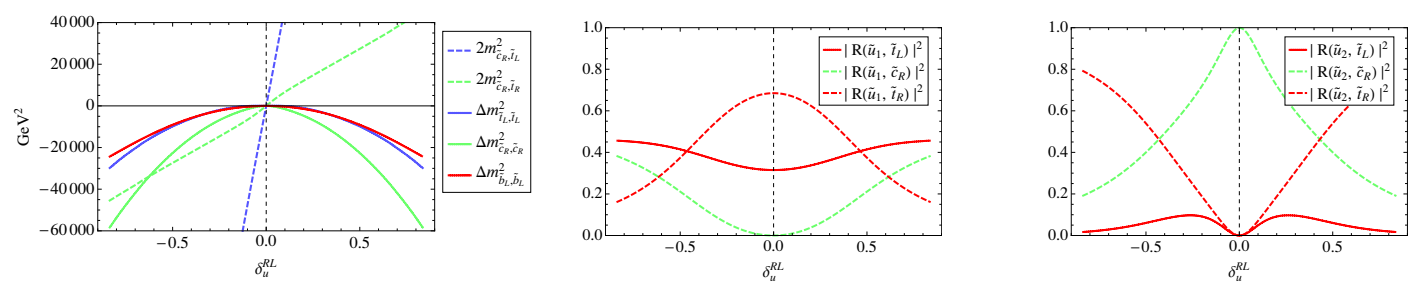

Figure 6. Dependence of up-type squark masses (left) and flavour decompositions of selected downtype squarks (centre and right) on the NMFV-parameter $\delta_{u}^{R L}$ based on the benchmark scenario SPS1a'. The point $\delta_{u}^{R L}=0$ corresponds to the MFV-scenario of table 2 .

not only imply important corrections to the couplings as discussed above, but can also lead to new flavour-mixing entries that are induced at the one-loop level.

We first discuss the parameter $\delta_{u}^{R L}$ that induces the one-loop contributions to $\tilde{c}_{R}-\tilde{t}_{R}$ mixing shown in figure 5. Here, the contribution from the light Higgs-boson $h^{0}$ is more important since in the up-squark sector in the decoupling limit the couplings to the heavy Higgs bosons are $\tan \beta$-suppressed. Another difference w.r.t. the sdown-mixing parameters $\delta_{d}^{R L, L R}$ is that the loop-induced right-right mixing becomes relevant because of the large value of $\left(\hat{T}_{U}\right)_{33}$ which enters the loop via the combination $\left(\hat{T}_{U}\right)_{32}\left(\hat{T}_{U}\right)_{33}$. The corrections to the mass parameters, however, are due to the linear dependence much smaller than in the sdown-sector.

In order to numerically illustrate this, we show in figure 6 the mass parameters and selected entries of the mixing matrix as a function of the NMFV-parameter $\delta_{u}^{R L}$. The graph also shows that both mixing elements depend linearly on $\delta_{u}^{R L}$. The quadratically dependent self-energies cannot challenge the left-right mixing, so that $\tilde{u}_{1}$ and $\tilde{u}_{6}$ approach maximally mixed states consisting of $\tilde{c}_{R}$ and $\tilde{t}_{L}$ for $\delta_{u}^{R L} \rightarrow \pm 1$ whereas $\tilde{u}_{1}$ is a nearly pure $\tilde{t}_{R}$ state in this limit with a small $\tilde{c}_{R}$ admixture.

Finally, let us study variations of parameter $\delta_{u}^{L R}$ inducing a direct mixing between the gauge eigenstates $\tilde{c}_{L}$ and $\tilde{t}_{R}$. At the one-loop level, this parameter induces corrections to the mass parameter of $\tilde{s}_{L}$, the isospin partner of the left-handed flavour $\tilde{c}_{L}$. On top of that, the graphs shown in figure 7 lead to a sizeable mixing between $\tilde{c}_{L}$ and $\tilde{t}_{L}$ and between $\tilde{s}_{L}$ and $\tilde{b}_{L}$, the latter again due to the strong top Yukawa coupling. In contrast, the rather small charm Yukawa coupling does not allow for remarkable influence on the sector of sdown-squarks. This is also in contrast to the variation of $\delta_{u}^{R L}$ discussed above, where the opposite situation has been observed.

The diagonal mass parameters shown in figure 8 are again quadratic in the NMFVparameter $\delta_{u}^{L R}$, while the tree-level $\tilde{c}_{L}-\tilde{t}_{R}$ mixing and the loop-induced $\tilde{s}_{L}-\tilde{b}_{L}$ mixing show a linear dependence. The latter also holds asymptotically for the $\tilde{c}_{L}-\tilde{t}_{L}$ mixing, where the 

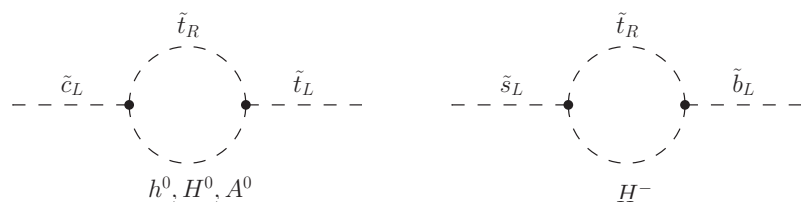

Figure 7. One-loop contributions to $\tilde{c}_{L}-\tilde{t}_{L}$ and $\tilde{s}_{L}-\tilde{b}_{L}$ mixing that are linear in the parameter $\delta_{u}^{L R}$.
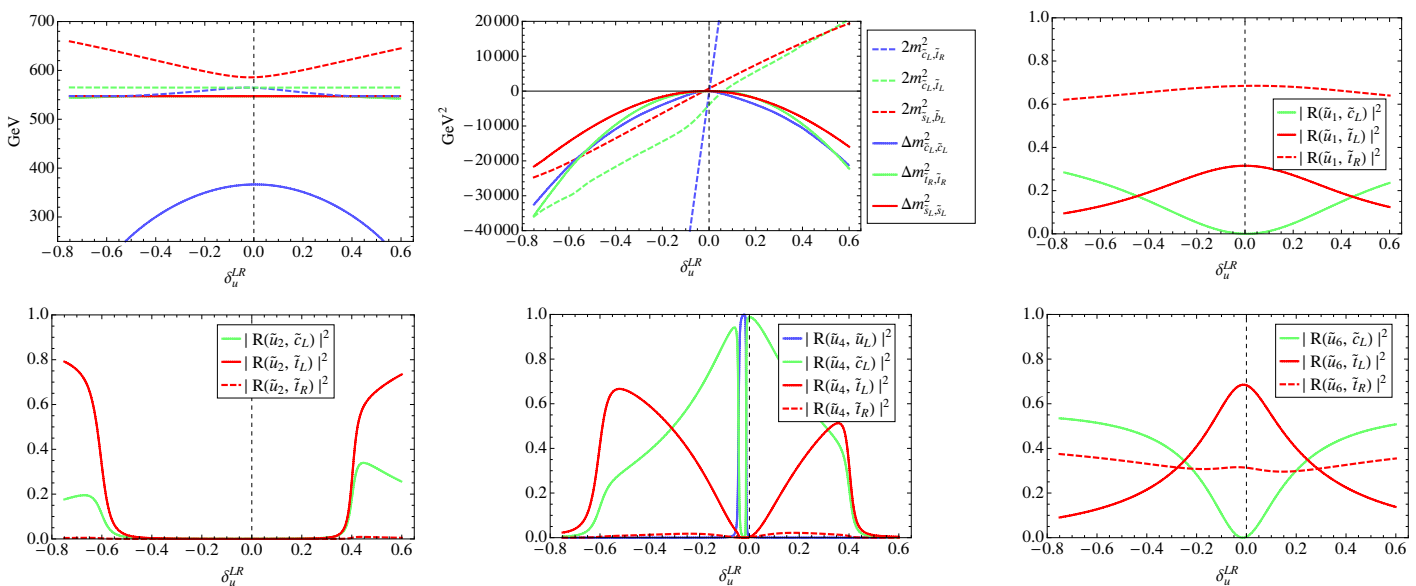

Figure 8. Dependence of physical squark masses (top left), one-loop contributions to squark mass parameters (top centre) and flavour decompositions of selected up-type squarks (top right and bottom) on the NMFV-parameter $\delta_{u}^{L R}$ based on the benchmark scenario SPS1a'. The point $\delta_{u}^{L R}=0$ corresponds to the MFV-scenario of table 2 .

offset can be traced to the fact that the left-left block of the up-type squark mass matrices is not diagonal in the super-CKM basis. This effect is also visible in a slight asymmetry in the flavour contents shown in figure 8 . The level-crossing between $\tilde{u}_{2}$ and $\tilde{u}_{4}$ occurs, e.g., at $\delta_{u}^{L R} \approx-0.6$ and $\delta_{u}^{L R} \approx 0.4$.

We also see in figure 8 that the decrease of the diagonal elements cannot compensate the increase of the $\tilde{c}_{L}-\tilde{t}_{R}$ mixing. In consequence, we observe a rather "conventional" mass splitting tending towards a equipartition of the mixing flavours in the lightest and heaviest mass eigenstates $\tilde{u}_{1}$ and $\tilde{u}_{6}$ for $\delta_{u}^{L R} \rightarrow \pm 1$.

\section{Quark flavour violating decays of squarks into bosons}

Assuming MFV, the couplings of squarks to $Z^{0}$ - and Higgs-bosons are in good approximation diagonal in generation space. In consequence, only two squark flavours of the same generation can be involved in the corresponding interactions. As a further consequence, the decay of a given squark $\tilde{q}_{i}$ into a $Z^{0}$ - or Higgs-boson can involve only one further squark $\tilde{q}_{j}$ (if kinematically allowed). For our reference scenarios, this can be seen in table 3 , where we show the branching ratios of the kinematically allowed decays of squarks into gauge and Higgs-bosons. Note that in both cases the heavier $u$-squark is mainly the heavier stop.

In the same way, decays of a certain down- (up-)squark $\tilde{d}_{i}\left(\tilde{u}_{i}\right)$ into $W^{ \pm}$-bosons can involve maximally two up- (down-)squarks $\tilde{d}_{j}\left(\tilde{u}_{j}\right)$ and $\tilde{d}_{k}\left(\tilde{u}_{k}\right)$ as final states. For our exam- 


\begin{tabular}{|c|cc|}
\hline SPS1a' & \multicolumn{2}{|c|}{ Branching ratios } \\
\hline$W^{ \pm}$ & $\tilde{d}_{1} \rightarrow \tilde{u}_{1} W^{-}$ & $30.5 \%$ \\
& $\tilde{d}_{2} \rightarrow \tilde{u}_{1} W^{-}$ & $40.2 \%$ \\
\hline$Z^{0}$ & $\tilde{u}_{6} \rightarrow \tilde{u}_{1} Z^{0}$ & $36.4 \%$ \\
\hline$h^{0}$ & $\tilde{u}_{6} \rightarrow \tilde{u}_{1} h^{0}$ & $5.5 \%$ \\
\hline
\end{tabular}

\begin{tabular}{|c|cc|}
\hline SPS1b & \multicolumn{2}{|c|}{ Branching ratios } \\
\hline$W^{ \pm}$ & $\tilde{d}_{1} \rightarrow \tilde{u}_{1} W^{-}$ & $3.6 \%$ \\
& $\tilde{d}_{2} \rightarrow \tilde{u}_{1} W^{-}$ & $13.6 \%$ \\
\hline$Z^{0}$ & $\tilde{u}_{2} \rightarrow \tilde{u}_{1} Z^{0}$ & $9.6 \%$ \\
\hline$h^{0}$ & $\tilde{u}_{2} \rightarrow \tilde{u}_{1} h^{0}$ & $3.0 \%$ \\
\hline
\end{tabular}

Table 3. Branching ratios of kinematically allowed decays of squarks into gauge and Higgs bosons for the reference scenarios SPS1a' (left) and SPS1b (right) assuming MFV.

ples, we have the decays $\tilde{d}_{1} \rightarrow \tilde{u}_{1} W^{-}$and $\tilde{d}_{2} \rightarrow \tilde{u}_{1} W^{-}$. Note that due to the CKM-matrix further flavour-violating decays are in principal allowed, e.g. due to a $\tilde{s}_{L} \rightarrow \tilde{t}_{L}$ transition. These are, however, strongly suppressed w.r.t. to the generation-conserving channels mentioned above. The generation-conserving transitions $\tilde{u}_{6} \rightarrow \tilde{d}_{1,2} W^{+}$are kinematically forbidden in the SPS1a' scenario.

Let us now discuss which experimental signatures related to squark decays would be able to challenge the hypothesis of MFV. If at least one of the NMFV-parameters in eq. (2.6) is non-zero, a further up-type squark mass eigenstate can obtain sufficiently large admixtures of $\tilde{t}_{R}$ or $\tilde{t}_{L}$ in order to open a new decay channel with a $Z^{0}$ or $h^{0}$ in the final state. In addition, the modified mass splitting alters the kinematical conditions as discussed in section 3. In the same way, non-vanishing generation mixing can allow for more than one (two) squark(s) decaying into the same final state squark and a neutral (charged) boson. Observing such additional decays would be a clear hint towards a nonminimal flavour structure in the squark sector. Obviously, the same reasoning also holds for the down-type squarks.

To summarise, relevant NMFV-signatures for decays of squarks into final states with $W^{ \pm}, Z^{0}$, and Higgs-bosons would be:

- $\tilde{q}_{i} \rightarrow \tilde{q}_{j} Z^{0}$ or $\tilde{q}_{i} \rightarrow \tilde{q}_{j} h^{0}$ for a fixed value of $i$ and at least two different values of $j$ or for a fixed value of $j$ and at least two different values of $i$,

- $\tilde{q}_{i} \rightarrow \tilde{q}_{j}^{\prime} W^{ \pm}$or $\tilde{q}_{i} \rightarrow \tilde{q}_{j}^{\prime} H^{ \pm}$for a fixed value of $i$ and at least three different values of $j$ or for a fixed value of $j$ and at least three different values of $i$.

In the following, we discuss our results for the scenarios SPS1a' and SPS1b for the variation of one or two NMFV-parameters.

\subsection{Signatures for the variation of one NMFV-parameter}

As our main reference scenario for the following discussion we choose again the benchmark point SPS1a'. For specific cases, we shall also comment on SPS1b. The experimental constraints of table 1 allow for rather large variations of the NMFV-parameter $\delta_{d}^{R R}$. The strongest constraint is here imposed by the observable $\Delta m_{B_{s}}$, leading to an allowed range of $-0.36 \lesssim \delta_{d}^{R R} \lesssim 0.69$. The constraint from $b \rightarrow s \gamma$ is less stringent in this case. In the left panel of figure 9, we show selected branching ratios of down-type squarks into 

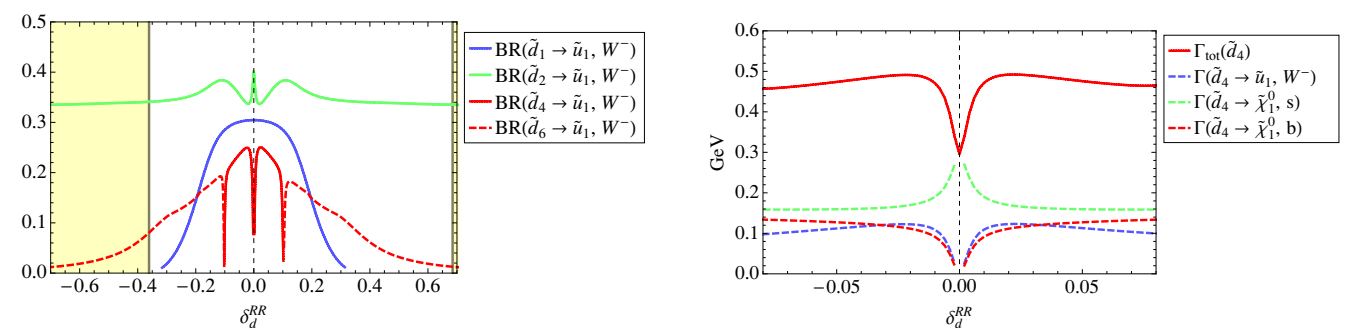

Figure 9. Branching ratios of squarks decaying into W-bosons (left) and total and partial decay widths of $\tilde{d}_{4}$ (right) for variations of $\delta_{d}^{R R}$ around the reference scenario SPS1a'. The shaded region indicates the experimentally disfavoured points.

W-bosons in dependence of this NMFV-parameter. The shaded regions correspond to the experimentally disfavoured parameter configurations. In the case of $\mathrm{MFV}$ (i.e. $\delta_{d}^{R R}=0$ ), only the decays of $\tilde{d}_{1}$ and $\tilde{d}_{2}$ into $\tilde{u}_{1}$ are possible (see table 3 ). However, already for small variations of $\delta_{d}^{R R}$, the small $\tilde{b}_{L}$ content in $\tilde{d}_{4}$ is sufficient to open the additional decay channel $\tilde{d}_{4} \rightarrow \tilde{u}_{1} W^{-}$with a comparable branching ratio.

The right panel of figure 9 shows that the rapid increase of the branching fraction is due to the increase of the partial width of this particular channel combined with a decrease of the decay $\tilde{d}_{4} \rightarrow \tilde{\chi}_{1}^{0} s$, which is dominant in the MFV-case. For small values of $\delta_{d}^{R R}$, the decrease of the coupling strength is compensated by the increased mass splitting, so that the width $\Gamma\left(\tilde{d}_{4} \rightarrow \tilde{u}_{1} W^{-}\right)$remains practically constant. For SPS1a' we observe thus a strong NMFV-signature with three important branching ratios around $30 \%$ related to decays into a W-boson for $\delta_{d}^{R R} \lesssim \pm 0.1$. For SPS1b, the channels involving W-bosons are less important, reaching branching ratios from $3 \%$ to $13 \%$.

Since the parameter $\delta_{d}^{R R}$ has no influence on the sector of up-type squarks, no further decay of an up-squark into a $Z^{0}$ - or Higgs-bosons can be induced. In the MFV-case (see table 3), the mass splitting is not sufficient to allow for such a decay for down-type squarks. For increasing flavour mixing, the decay of a down-type squark into a neutral bosons becomes possible allowed due to the increased mass splitting. For $\delta_{d}^{R R} \gtrsim 0.2(0.25)$, e.g., the decay $\tilde{d}_{6} \rightarrow \tilde{d}_{1} Z^{0}\left(\tilde{d}_{4,5} \rightarrow \tilde{d}_{1} Z^{0}\right)$ is kinematically allowed. For higher $\delta_{d}^{R R}$, also decays into $h^{0}$ would be possible. However, the necessary $\tilde{b}_{L}$ and $\tilde{s}_{L}$ contents are decreasing at the same time, so that the corresponding coupling is suppressed. For similar reasons, kinematically allowed decays into $h^{0}$ cannot be observed since the coupling is approximately zero due to a negative interference between the D- and F-term contributions.

Variations of the parameter $\delta_{u}^{R R}$ only influence the sector of up-type squarks in such a way that no NMFV-signatures with decays of sdown-squarks into W-bosons can be induced. There are only the two possible modes given in table 3. Nevertheless, the corresponding branching ratios depend on the flavour-mixing parameter $\delta_{u}^{R R}$ due to decreasing mass of $\tilde{u}_{1}$. At the same time, the mass of $\tilde{u}_{6}$ increases, so that the decay $\tilde{u}_{6} \rightarrow \tilde{d}_{1} W^{+}$opens for $\left|\delta_{u}^{R R}\right| \gtrsim 0.15$. However, this decay cannot by interpreted as a typical NMFV-signal within this context. The allowed range for this NMFV-parameter is $-0.8 \lesssim \delta_{u}^{R R} \lesssim 0.8$, where the main constraints come from squark mass limits rather than the observables of table 1.

Such signatures, however, can occur in the context of decays involving Z- or Higgs- 

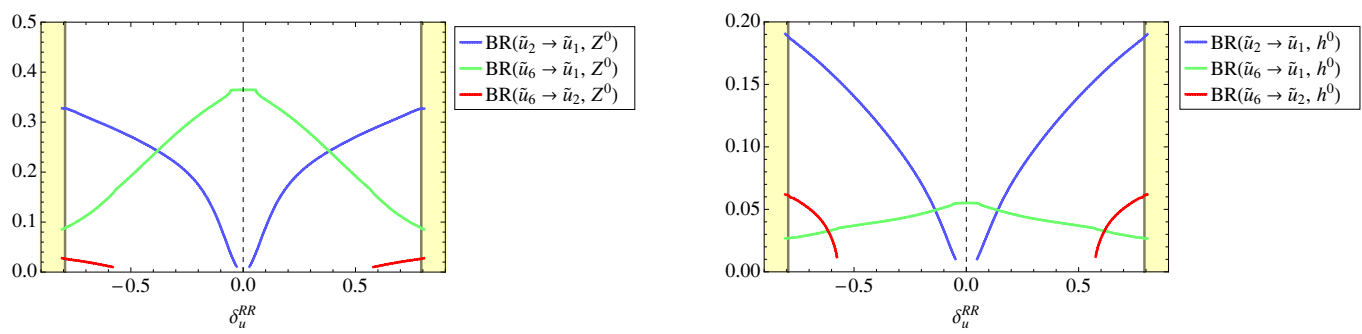

Figure 10. Branching ratios of squarks decaying into Z- (left) and Higgs-bosons (right) for variations of $\delta_{u}^{R R}$ around the reference scenario SPS1a'. The shaded region indicates the experimentally disfavoured points.
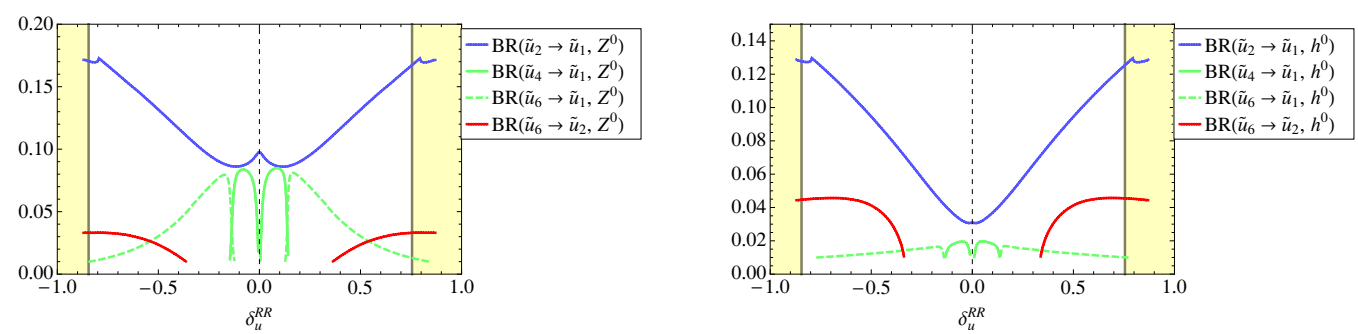

Figure 11. Branching ratios of squarks decaying into Z- (left) and Higgs-bosons (right) for variations of $\delta_{u}^{R R}$ around the reference scenario SPS1b. The shaded region indicates the experimentally disfavoured points.

bosons. In figure 10 we show the corresponding branching ratios of up-type squarks. While for $\delta_{u}^{R R}=0$, only one such decay is possible, additional channels open with sizeable branching fractions already for moderate flavour mixing. In particular, there are three states containing $\tilde{t}_{L}$, and the mass splitting creates sufficient phase-space to produce an on-shell Z- or Higgs-boson. As can be seen from figure 10, basically the whole range of $0.02<\left|\delta_{u}^{R R}\right|<0.8$ allows for the NMFV-typical signature of the two up-squarks $\tilde{u}_{2}$ and $\tilde{u}_{6}$ decaying into $\tilde{u}_{1}$ and $Z^{0}$ or $h^{0}$. For $\left|\delta_{u}^{R R}\right| \gtrsim 0.5$, even a second NMFV-signature shows up, namely the additional decays $\tilde{u}_{6} \rightarrow \tilde{u}_{2} Z^{0}\left(h^{0}\right)$ reaching branching ratios of up to 0.02 (0.07).

For comparison, we show in figure 11 the corresponding branching ratios of up-type squarks for the reference scenario SPS1b. Generally, this point features lower branching ratios. It is interesting to note that there is even a small range around $\delta_{u}^{R R} \approx 0.138$, where the decays of three different squarks into the same final state are possible. This feature is associated to a rather smooth level-crossing between $\tilde{u}_{4}$ and $\tilde{u}_{6}$, where both states have a sizeable $\tilde{t}_{L}$-content. The main difference w.r.t. SPS1a' lies in the initial mass splitting in the MFV-case, which is not sufficient to make $\tilde{t}_{2}$ the heaviest up-type squark (see table 2). Instead the third generation mass eigenstates are the two lightest ones, as is also the case for the down-type squarks. This leads to a different structure of the flavour mixings induced by the NMFV-parameters.

In contrast to the flavour mixing in the right-right sectors discussed above, the parameter $\delta^{L L}$ is rather constrained due to the decay $b \rightarrow s \gamma$, allowing only for the narrow interval $-0.08 \lesssim \delta^{L L} \lesssim 0.03$. A second allowed window around $\delta^{L L} \approx 0.8$, where the large 
SUSY contributions to $\operatorname{BR}(b \rightarrow s \gamma)$ cancel, is disfavoured by both $\Delta m_{B_{s}}$ and $\bar{B}_{s} \rightarrow l^{+} l^{-}$. Concerning NMFV-signatures, three decay channels of down-type squarks into a W-boson and the lightest up-type squark are open for $\left|\delta^{L L}\right| \gtrsim 0.04$. Further signal channels would open beyond the experimental exclusion limit. In the same way, the two decay channels $\tilde{u}_{4} \rightarrow \tilde{u}_{1} Z^{0}$ and $\tilde{u}_{6} \rightarrow \tilde{u}_{1} Z^{0}$ lead to a weak NMFV-signature where the additional decays reach at most $10 \%$ at the boundary of the experimentally allowed range. Again, further decay modes would be allowed outside the experimentally favoured interval for the parameter $\delta^{L L}$. The branching ratios of squark decays into Higgs-bosons are significantly smaller because the corresponding couplings get small for large left-right mixing.

Apart from the effects on the squark mass eigenvalues discussed in section 3, variations of the NMFV-parameter in the off-diagonal blocks of eq. (2.1) can also induce the characteristic signatures in the decays of squarks. Flavour mixing in this sector of down-type squarks is mainly constrained from the experimental limit on the meson-oscillation observable $\Delta m_{B_{s}}$ and the branching ratio of $b \rightarrow s \gamma$, which leave only the rather narrow interval of $-0.02 \lesssim \delta_{d}^{R L} \lesssim 0.15$. The experimentally allowed ranges for the parameter $\delta_{d}^{L R}$ are even more narrow. Here, the constraint from $b \rightarrow s \gamma$ only allows for $-0.005 \lesssim \delta_{d}^{L R} \lesssim 0.004$. Due to the small allowed ranges, we do not discuss the variation of the parameters $\delta_{d}^{R L}$ and $\delta_{d}^{L R}$ in detail. In particular, the latter does not lead to NMFV-signatures for our reference scenarios SPS1a' and SPS1b. For SPS1a' and variations of $\delta_{d}^{R L}$, sizeable branching ratios $\operatorname{BR}\left(\tilde{d}_{1} \rightarrow \tilde{u}_{1} W^{+}\right) \approx \operatorname{BR}\left(\tilde{d}_{2} \rightarrow \tilde{u}_{1} W^{+}\right) \approx \operatorname{BR}\left(\tilde{d}_{3} \rightarrow \tilde{u}_{1} W^{+}\right) \approx 20-30 \%$ can be observed around $\delta_{d}^{R L} \approx-0.016$ and $\delta_{d}^{R L} \approx 0.013$, in direct vicinity of the exclusion due to the constraint from $b \rightarrow s \gamma$ and $\Delta M_{B_{s}}$. Additional decay channels involving Z- or Higgs-bosons are not opened within this range. The same qualitative picture is obtained for SPS1b.

Again, the most interesting parameters are the ones leading to NMFV-signatures with neutral bosons. They allow for rather large experimentally allowed ranges $-0.39 \lesssim \delta_{u}^{R L} \lesssim$ 0.39 and $-0.29 \lesssim \delta_{u}^{L R} \lesssim 0.10$. Interestingly, the limit on $\delta_{u}^{R L}$ comes not from the low-energy observables, but from the experimental limit on the mass of the lightest Higgs-boson. The latter becomes lighter for increasing flavour mixing due to the corrections from squarkloops. For $\delta_{u}^{L R}$, the decay $b \rightarrow s \gamma$ remains the most stringent constraint.

Let us start the discussion with the parameter $\delta_{u}^{L R}$ inducing a $\tilde{c}_{R}-\tilde{t}_{L}$ mixing. Here, additional decays into $\mathrm{W}$-bosons cannot be achieved due to the fact that, except $\tilde{d}_{1}$ and $\tilde{d}_{6}$, no down-type squarks obtain a $\tilde{b}_{L}$ admixture, which can lead to decays into $\tilde{u}_{1}$. In the case of MFV, the decay $\tilde{u}_{6} \rightarrow \tilde{u}_{1} Z^{0}$ is the only channel involving a Z-boson. For already rather small $\left|\delta_{u}^{R L}\right| \gtrsim 0.03$, a second channel $\tilde{u}_{2} \rightarrow \tilde{u}_{1} Z^{0}$ with the same final state opens with a branching ratio of up to about $10 \%$ as shown in figure 12 . The reason therefore is the non-zero $\tilde{t}_{L}$ content in $\tilde{u}_{2}$ (see figure 6). Its decrease for $\left|\delta_{u}^{R L}\right| \gtrsim 0.25$ is compensated by the increasing phase space so that the branching ratio remains nearly constant. The branching ratio of the initial decay $\tilde{u}_{6} \rightarrow \tilde{u}_{1} Z^{0}$ increases with the mass splitting. The corresponding coupling only changes within a range of $10 \%$.

In figure 12, we show the decays of squarks into light Higgs bosons as a function of the NMFV-parameter $\delta_{u}^{R L}$ for the reference scenario SPS1a'. Here, the initial decay $\tilde{u}_{6} \rightarrow \tilde{u}_{1} h^{0}$ decreases with increasing flavour mixing. The relevant coupling is dominated by a left-right component, but the $\tilde{t}_{R}$ content of $\tilde{u}_{1}$ and $\tilde{u}_{6}$ decreases (see figure 6 ). For $\left|\delta_{u}^{R L}\right| \gtrsim 0.3$, the 

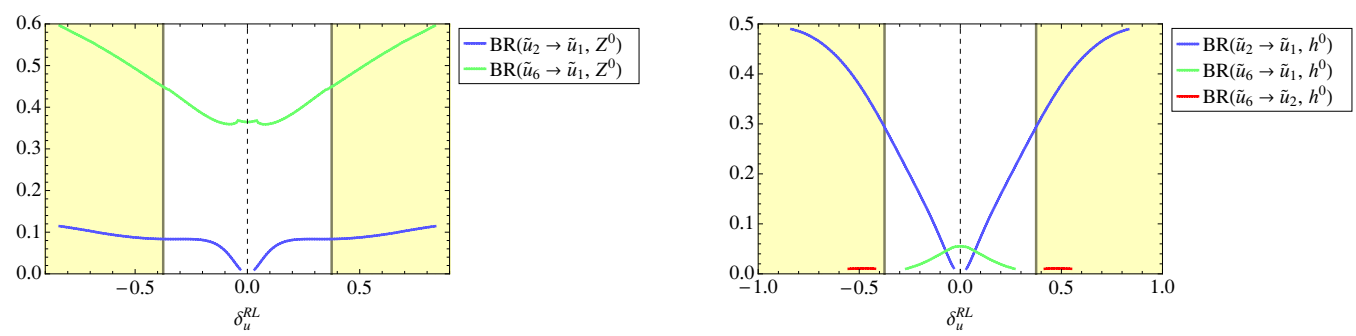

Figure 12. Branching ratios of squarks decaying into Z- (left) and Higgs-bosons (right) for variations of $\delta_{u}^{R L}$ around the reference scenario SPS1a'. The shaded region indicates the experimentally disfavoured points.

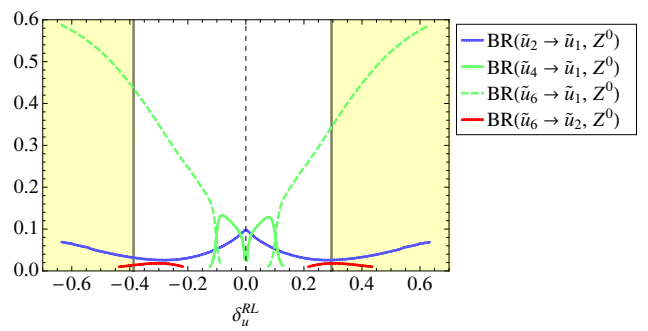

Figure 13. Branching ratios of squarks decaying into Z-bosons for variations of $\delta_{u}^{R L}$ around the reference scenario SPS1b. The shaded region indicates the experimentally disfavoured points.

new channel $\tilde{u}_{2} \rightarrow \tilde{u}_{1} h^{0}$ opens leading to a characteristic NMFV-signature. However, the decay $\tilde{u}_{6} \rightarrow \tilde{u}_{1} h^{0}$ falls rapidly below $1 \%$, so that only the rather narrow interval $-0.03 \leq \delta_{u}^{R L} \leq 0.18$ allows for a realistic signature.

In the case of SPS1b, variations of the parameter $\delta_{u}^{R L}$ qualitatively show the same signature. However, the different mass splitting and the associated smooth level-crossing between $\tilde{u}_{4}$ and $\tilde{u}_{6}$ at $\delta_{u}^{R L} \approx \pm 0.1$ lead to a small interval around this point where three states $\tilde{u}_{2,4,6}$ can decay into the same final state $\tilde{u}_{1} Z^{0}$. This behaviour is analogous to the case of $\delta_{u}^{R R}$ discussed above. The numerical results for the branching ratios of these decays are shown in figure 13 .

Finally, let us discuss the squark decays as a function of the parameter $\delta_{u}^{L R}$. For this parameter, it is again possible to observe a NMFV-signature from decays into Wbosons. In figure 14, we show examples of numerical branching ratios with the lightest up-type squark and a W-boson in the final state for our two reference scenarios. While around $\delta_{u}^{L R} \approx 0$ only the decays already mentioned in table 3 are allowed for SPS1a', a third channel $\tilde{d}_{5} \rightarrow \tilde{u}_{1} W^{+}$opens for $\delta_{u}^{L R} \lesssim-0.1$ and $\delta_{u}^{L R} \gtrsim 0.08$. The corresponding branching ratio amounts to up to $20 \%$ within the experimentally allowed range, leading to a sizeable NMFV-signature. Note that this signature is not typical for a parameter in the sector of up-squarks and is possible only due to the loop-induced $\tilde{s}_{L}-\tilde{b}_{L}$ mixing discussed in section 3. Due to the mass splitting, the three relevant branching ratios increase with the flavour-mixing parameter.

For SPS1b, the branching ratios of the three signal decays $\tilde{d}_{1,2,3} \rightarrow \tilde{u}_{1} W^{+}$are with branching ratios of about $30-60 \%$ rather sizeable. For $\delta_{u}^{L R} \gtrsim-0.35$, even a fourth channel 

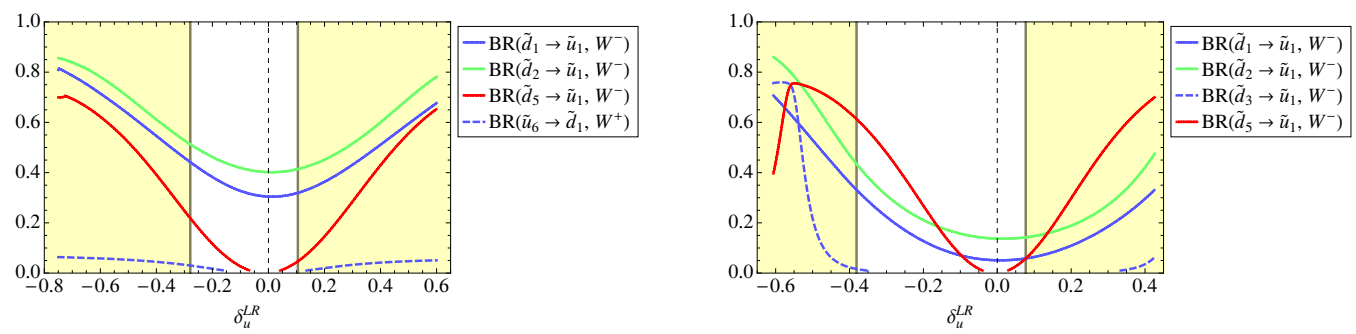

Figure 14. Branching ratios of squarks decaying into W-bosons for variations of $\delta_{u}^{L R}$ around the reference scenarios SPS1a' (left) and SPS1b (right). The shaded region indicates the experimentally disfavoured points.

due to the decay of $\tilde{d}_{5}$ opens up. However, the corresponding branching fraction in the experimentally allowed range remains below $1.6 \%$.

The above results for SPS1a' and SPS1b can be summarized as follows:

- The NMFV-parameters in the sdown-sector only induce signatures related to Wbosons.

- The parameters in the sup-sector induce mainly signatures related to Z- and Higgsbosons. The latter have mostly smaller branching ratios due to the suppressed phase space.

- The parameter $\delta^{L L}$ acts on both the sup- and sdown-sectors and can therefore induce three W-channels as well as two Z- or Higgs-channels. However, this parameter is heavily constraint from experimental data.

- The parameters $\delta_{u}^{R R}$ and $\delta_{d}^{R R}$ are rather unconstrained and allow for large mass splitting leading to large branching ratios for decays into vector bosons.

These features are to some extend a consequence of the original mSUGRA boundary conditions. However, the basic structure is the same as in other breaking schemes like GMSB or AMSB. One would need some non-universal boundary condition at the high scale to depart from this feature. One possibility are, e.g., extra $D$-term contributions occurring from the breaking of a higher rank group to the SM group. For example, if $\mathrm{SO}(10)$ or $E(6)$ get broken to the SM, there are $D$-terms contributing differently to the left- and right squarks [35].

Finally, selected signatures for the variation of one single NMFV-parameter for the reference scenarios SPS1a' and SPS1b are collected in tables 4 and 5, respectively. They have been chosen by the requirement to provide potentially large signals.

\subsection{Signatures for the variation of two NMFV-parameters}

The signatures related to non-minimal flavour violation in the squark sector discussed above can be extended or amplified if more than one of the parameters defined in eq. (2.6) is non-zero. In particular, the relevant branching ratios can be increased and additional NMFV-signatures can appear. Here, we limit ourselves to the simultaneous variation of 


\begin{tabular}{|c|c|}
\hline \multicolumn{3}{|c|}{ SPS1a' } \\
\hline$\delta_{d}^{R R}= \pm 0.016$ & $\delta_{u}^{R R}= \pm 0.75$ \\
$\operatorname{BR}\left(\tilde{d}_{1} \rightarrow \tilde{u}_{1} W^{-}\right)=30 \%$ & $\operatorname{BR}\left(\tilde{u}_{2} \rightarrow \tilde{u}_{1} Z^{0}\right)=32 \%$ \\
$\operatorname{BR}\left(\tilde{d}_{2} \rightarrow \tilde{u}_{1} W^{-}\right)=34 \%$ & $\operatorname{BR}\left(\tilde{u}_{6} \rightarrow \tilde{u}_{1} Z^{0}\right)=9.8 \%$ \\
$\operatorname{BR}\left(\tilde{d}_{4} \rightarrow \tilde{u}_{1} W^{-}\right)=25 \%$ & $\operatorname{BR}\left(\tilde{u}_{6} \rightarrow \tilde{u}_{2} Z^{0}\right)=2.4 \%$ \\
& $\operatorname{BR}\left(\tilde{u}_{2} \rightarrow \tilde{u}_{1} h^{0}\right)=18 \%$ \\
& $\operatorname{BR}\left(\tilde{u}_{6} \rightarrow \tilde{u}_{1} h^{0}\right)=2.8 \%$ \\
& $\operatorname{BR}\left(\tilde{u}_{6} \rightarrow \tilde{u}_{2} h^{0}\right)=5.7 \%$ \\
\hline$\delta^{L L}=-0.06$ & $\delta_{u}^{L R}=-0.25$ \\
$\operatorname{BR}\left(\tilde{d}_{1} \rightarrow \tilde{u}_{1} W^{-}\right)=29 \%$ & $\operatorname{BR}\left(\tilde{d}_{1} \rightarrow \tilde{u}_{1} W^{-}\right)=42 \%$ \\
$\operatorname{BR}\left(\tilde{d}_{2} \rightarrow \tilde{u}_{1} W^{-}\right)=33 \%$ & $\operatorname{BR}\left(\tilde{d}_{2} \rightarrow \tilde{u}_{1} W^{-}\right)=49 \%$ \\
$\operatorname{BR}\left(\tilde{d}_{6} \rightarrow \tilde{u}_{1} W^{-}\right)=3.2 \%$ & $\operatorname{BR}\left(\tilde{d}_{5} \rightarrow \tilde{u}_{2} W^{-}\right)=18 \%$ \\
$\operatorname{BR}\left(\tilde{u}_{4} \rightarrow \tilde{u}_{1} Z^{0}\right)=5.4 \%$ & $\operatorname{BR}\left(\tilde{u}_{4} \rightarrow \tilde{u}_{1} Z^{0}\right)=2.4 \%$ \\
$\operatorname{BR}\left(\tilde{u}_{6} \rightarrow \tilde{u}_{1} Z^{0}\right)=30 \%$ & $\operatorname{BR}\left(\tilde{u}_{6} \rightarrow \tilde{u}_{1} Z^{0}\right)=39 \%$ \\
\hline$\delta_{u}^{R L}= \pm 0.15$ & $\delta_{d}^{R L}=-0.016$ \\
$\operatorname{BR}\left(\tilde{u}_{2} \rightarrow \tilde{u}_{1} Z^{0}\right)=7.4 \%$ & $\operatorname{BR}\left(\tilde{d}_{1} \rightarrow \tilde{u}_{1} W^{-}\right)=30.0 \%$ \\
$\operatorname{BR}\left(\tilde{u}_{6} \rightarrow \tilde{u}_{1} Z^{0}\right)=37 \%$ & $\operatorname{BR}\left(\tilde{d}_{2} \rightarrow \tilde{u}_{1} W^{-}\right)=27.0 \%$ \\
$\operatorname{BR}\left(\tilde{u}_{2} \rightarrow \tilde{u}_{1} h^{0}\right)=12 \%$ & $\operatorname{BR}\left(\tilde{d}_{3} \rightarrow \tilde{u}_{1} W^{-}\right)=34.0 \%$ \\
$\operatorname{BR}\left(\tilde{u}_{6} \rightarrow \tilde{u}_{1} h^{0}\right)=2.8 \%$ & \\
\hline
\end{tabular}

Table 4. Branching ratios of squark decays leading to typical NMFV-signatures for selected parameter points beyond MFV based on the benchmark scenario SPS1a'.

two NMFV-parameters, leaving more involved variations for later analyses. We also discuss only the combinations of parameters that lead to new signatures w.r.t. the variations discussed in section 4.1.

Since the above analysis has shown that the most interesting parameters are $\delta^{L L}$ and $\delta_{u}^{L R}$, we start our discussion with their simultaneous variation. A second interesting combination of parameters is $\delta_{u}^{L R}$ together with $\delta_{u}^{R R}$. The latter is experimentally rather unconstrained and leads to sizeable NMFV-signatures. In particular, it is the only parameter inducing a signature with two decays of the same initial state squark into different final state squarks and a light Higgs boson (see tables 4 and 5). Since $\delta_{u}^{R R}$ does not act on the the sector of down-type squarks, no signatures with W-bosons can be realized in the case of the single-parameter variation. However, taking a second NMFV-parameter to be non-zero can relax this limitation and open new signals.

In figure 15 we show the experimentally allowed ranges at the $95 \%$ confidence level in the $\delta^{L L}-\delta_{u}^{L R}$ and $\delta_{u}^{R R}-\delta_{u}^{L R}$-planes around our reference scenario SPS1a'. The decisive constraint is here again the inclusive decay $b \rightarrow s \gamma$, leading to a rather small interval for the 


\begin{tabular}{|c|c|c|c|c|c|}
\hline \multicolumn{6}{|c|}{ SPS1b } \\
\hline \multicolumn{3}{|c|}{$\delta_{d}^{R R}= \pm 0.027$} & \multicolumn{3}{|c|}{$\delta_{u}^{R R}= \pm 0.43$} \\
\hline \multirow{6}{*}{$\begin{array}{l}\operatorname{BR}\left(\tilde{d}_{1} \rightarrow \tilde{u}_{1} W^{-}\right) \\
\operatorname{BR}\left(\tilde{d}_{2} \rightarrow \tilde{u}_{1} W^{-}\right) \\
\operatorname{BR}\left(\tilde{d}_{4} \rightarrow \tilde{u}_{1} W^{-}\right)\end{array}$} & \multirow{6}{*}{$=$} & & $\operatorname{BR}\left(\tilde{u}_{2} \rightarrow \tilde{u}_{1} Z^{0}\right)$ & $=$ & $12 \%$ \\
\hline & & & $\operatorname{BR}\left(\tilde{u}_{6} \rightarrow \tilde{u}_{1} Z^{0}\right)$ & $=$ & $3.9 \%$ \\
\hline & & $6.8 \%$ & $\operatorname{BR}\left(\tilde{u}_{6} \rightarrow \tilde{u}_{2} Z^{0}\right)$ & $=$ & $1.7 \%$ \\
\hline & & & $\operatorname{BR}\left(\tilde{u}_{2} \rightarrow \tilde{u}_{1} h^{0}\right)$ & $=$ & $8.5 \%$ \\
\hline & & & $\operatorname{BR}\left(\tilde{u}_{6} \rightarrow \tilde{u}_{1} h^{0}\right)$ & $=$ & $1.3 \%$ \\
\hline & & & $\operatorname{BR}\left(\tilde{u}_{6} \rightarrow \tilde{u}_{2} h^{0}\right)$ & $=$ & $3.5 \%$ \\
\hline$\delta^{L L}=-0$ & & & $\delta_{u}^{L R}=-c$ & & \\
\hline $\operatorname{BR}\left(\tilde{d}_{1} \rightarrow \tilde{u}_{1} W^{-}\right)$ & $=$ & $4.6 \%$ & $\operatorname{BR}\left(\tilde{d}_{1} \rightarrow \tilde{u}_{1} W^{-}\right)$ & $=$ & $7.1 \%$ \\
\hline $\operatorname{BR}\left(\tilde{d}_{2} \rightarrow \tilde{u}_{1} W^{-}\right)$ & $=$ & $12 \%$ & $\operatorname{BR}\left(\tilde{d}_{2} \rightarrow \tilde{u}_{1} W^{-}\right)$ & $=$ & $16 \%$ \\
\hline $\operatorname{BR}\left(\tilde{d}_{6} \rightarrow \tilde{u}_{1} W^{-}\right)$ & $=$ & $1.9 \%$ & $\operatorname{BR}\left(\tilde{d}_{5} \rightarrow \tilde{u}_{1} W^{-}\right)$ & $=$ & $7.4 \%$ \\
\hline $\operatorname{BR}\left(\tilde{u}_{2} \rightarrow \tilde{u}_{1} Z^{0}\right)$ & $=$ & $8.2 \%$ & $\operatorname{BR}\left(\tilde{u}_{2} \rightarrow \tilde{u}_{1} Z^{0}\right)$ & $=$ & $8.0 \%$ \\
\hline $\operatorname{BR}\left(\tilde{u}_{6} \rightarrow \tilde{u}_{1} Z^{0}\right)$ & $=$ & $3.5 \%$ & $\operatorname{BR}\left(\tilde{u}_{6} \rightarrow \tilde{u}_{1} Z^{0}\right)$ & $=$ & $9.8 \%$ \\
\hline $\operatorname{BR}\left(\tilde{u}_{2} \rightarrow \tilde{u}_{1} h^{0}\right)$ & $=$ & $2.4 \%$ & $\operatorname{BR}\left(\tilde{u}_{2} \rightarrow \tilde{u}_{1} h^{0}\right)$ & $=$ & $1.7 \%$ \\
\hline $\operatorname{BR}\left(\tilde{u}_{6} \rightarrow \tilde{u}_{1} h^{0}\right)$ & $=$ & $1.2 \%$ & $\operatorname{BR}\left(\tilde{u}_{6} \rightarrow \tilde{u}_{1} h^{0}\right)$ & $=$ & $4.8 \%$ \\
\hline$\delta_{u}^{R L}= \pm 0$ & 102 & & & & \\
\hline $\operatorname{BR}\left(\tilde{u}_{2} \rightarrow \tilde{u}_{1} Z^{0}\right)$ & $=$ & $5.2 \%$ & & & \\
\hline $\operatorname{BR}\left(\tilde{u}_{4} \rightarrow \tilde{u}_{1} Z^{0}\right)$ & $=$ & $8.0 \%$ & & & \\
\hline $\operatorname{BR}\left(\tilde{u}_{6} \rightarrow \tilde{u}_{1} Z^{0}\right)$ & $=$ & $8.1 \%$ & & & \\
\hline
\end{tabular}

Table 5. Branching ratios of squark decays leading to typical NMFV-signatures for selected parameter points beyond MFV based on the benchmark scenario SPS1b.

left-left mixing parameter $\delta^{L L}$. The limits on the Higgs-mass and on $\Delta M_{B_{s}}$ are secondary. Non-zero values of $\delta_{u}^{L R}$ lead to small additional contributions to $\operatorname{BR}(b \rightarrow s \gamma)$ due to the loop-induced $\tilde{s}_{L}-\tilde{b}_{L}$ mixing, so that the different contributions cancel in certain regions of the $\delta^{L L}-\delta_{u}^{L R}$ plane. The experimentally allowed region is therefore slightly twisted w.r.t. the axes. The applied constraints allow for a large interval for the parameter $\delta_{u}^{R R}$, so that a rather wide concave favoured region is observed in the $\delta_{u}^{R R}-\delta_{u}^{L R}$-plane. For the second reference scenario SPS1b, the situation is qualitatively the same, with the exception that the vacuum stability [36] excludes certain regions in the $\delta_{u}^{R R}-\delta_{u}^{L R}$-plane that are allowed concerning the constraints of table 1 .

Let us now turn to the decays of squarks into W-bosons. Figure 16 shows that up to four decay channels can involve the same final state $\tilde{u}_{1} W^{-}$. The single graphs show the corresponding branching ratios in the $\delta^{L L}-\delta_{u}^{L R}$ plane. The main new feature compared to the aspects discussed in section 4.1 is that there is a wide experimentally allowed range where the channels $\tilde{d}_{6} \rightarrow \tilde{u}_{1} W^{-}$and $\tilde{d}_{5} \rightarrow \tilde{u}_{1} W^{-}$both are open. Note that this signature with up to four $\mathrm{W}$-bosons is possible in wide regions of parameter space. 

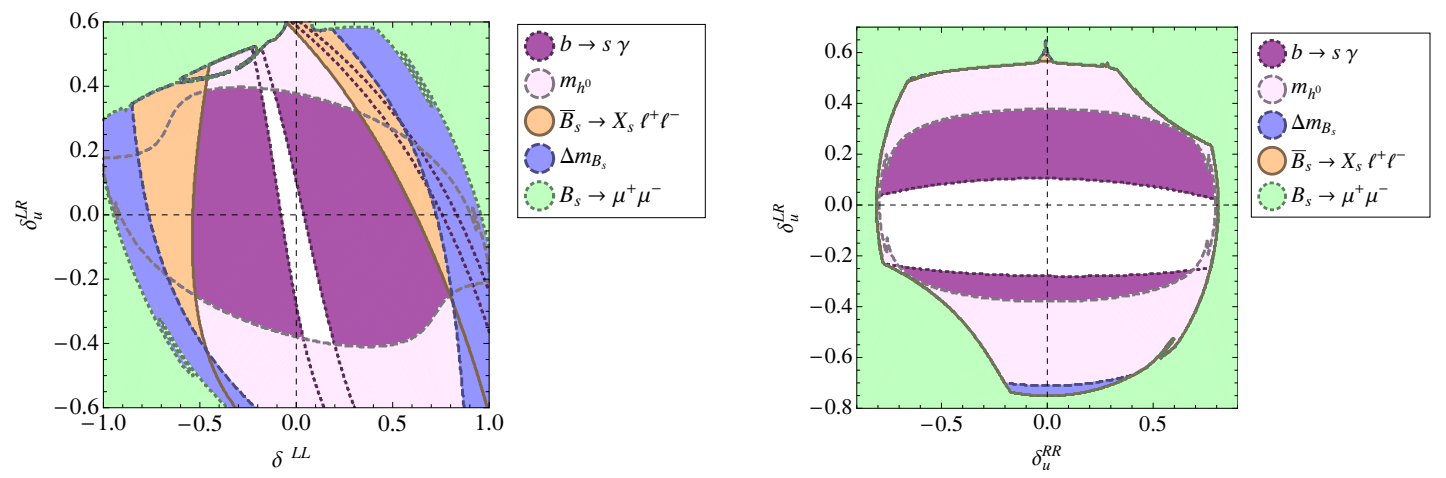

Figure 15. Experimental constraints for simultaneous variation of two NMFV-parameters around the reference scenario SPS1a'. The legend indicates the constraints in order of the excluded regions starting from the allowed white region.
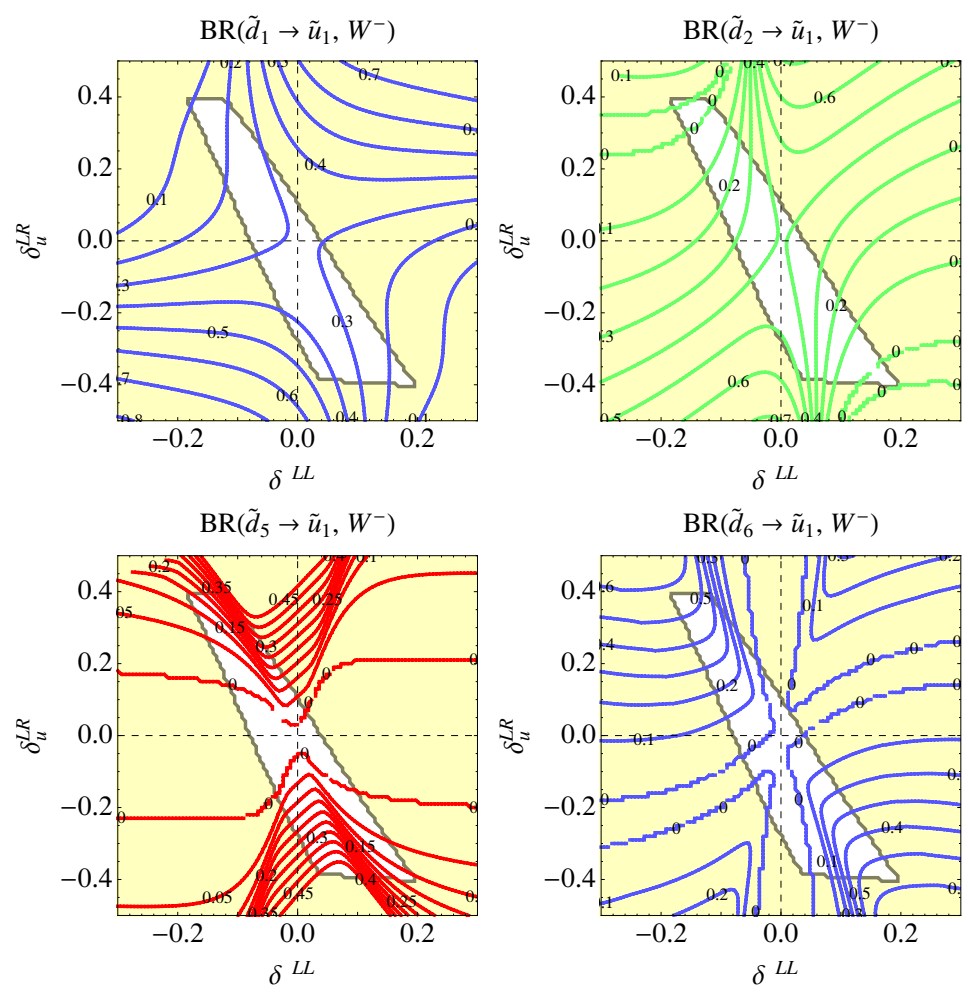

Figure 16. Dependence of the branching ratios of selected down-type squarks into the lightest uptype squark and a W-boson on the NMFV-parameters $\delta^{L L}$ and $\delta_{u}^{L R}$ around the reference scenario SPS1a'. The shaded region indicates the experimentally disfavoured points.

As discussed in the previous section, NMFV-signatures due to decays into Z-bosons are present over wide ranges of the analysed parameter space. However, there are only rather small regions where branching ratios for three different $u$-squarks into $\tilde{u_{1}} Z^{0}$ exceed five percent.

Concerning decays into light Higgs-bosons, the reference scenario SPS1a' does not lead to new features if one allows for two non-zero NMFV-parameters. Although the couplings 

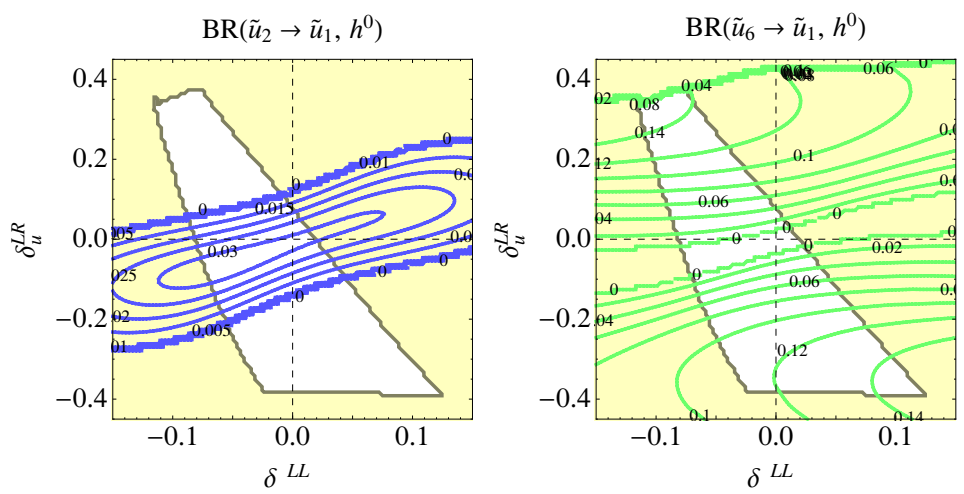

Figure 17. Dependence of the branching ratios of the down-type squarks $\tilde{u}_{2}$ and $\tilde{u}_{6}$ into the lightest up-type squark $\tilde{u}_{1}$ and a Higgs-boson $h^{0}$ on the NMFV-parameters $\delta^{L L}$ and $\delta_{u}^{L R}$ around the reference scenario SPS1b. The shaded region indicates the experimentally disfavoured points.

can become rather important, the phase space does not allow for additional branching ratios larger than 1\%. However, new decay channels involving Higgs-bosons open in the case of SPS1b, as can be seen in figure 17 . Here, we have $\tilde{u}_{6} \approx \tilde{c}_{L}$ if all NMFV-parameters are zero, and the two lightest squarks are the stops. For both parameters $\delta^{L L}$ and $\delta_{u}^{L R}$, the channel $\tilde{u}_{6} \rightarrow \tilde{u}_{1} h^{0}$ opens with branching ratios of up to almost $15 \%$. The branching fraction of the original decay channel $\tilde{u}_{2} \rightarrow \tilde{u}_{1} h^{0}$ (see table 3 ) decreases at the same time.

Finally, let us discuss NMFV-signatures related to a simultaneous variation of $\delta_{u}^{R R}$ and $\delta_{u}^{L R}$. For our scenario based on SPS1a', the branching ratios of up-type squarks into Zand Higgs-bosons are shown in figures 18 and 19, respectively. A large part of the twodimensional parameter space allows for three decay channels into the same final state $\tilde{u}_{1} Z^{0}$. The branching ratio $\operatorname{BR}\left(\tilde{u}_{2} \rightarrow \tilde{u}_{1} Z^{0}\right)$ increases mainly along the $\delta_{u}^{R R}$ direction, while it is almost independent of $\delta_{u}^{L R}$. This is explained by the fact that for MFV, $\tilde{u}_{2}$ is a pure $\tilde{c}_{R}$ state and no $\tilde{t}_{L}$ content is introduced for variations of $\delta_{u}^{L R}$. For the decay of $\tilde{u}_{4}$, the opposite is observed. This channel remains closed along the $\delta_{u}^{R R}$-axis, since this parameter mixes neither a $\tilde{t}_{L}$ content into $\tilde{u}_{4}$ nor a $\tilde{c}_{L}$ content into $\tilde{u}_{1}$. This only happens for $\left|\delta_{u}^{L R}\right|>0$. However, due to the destructive interference of the $\tilde{c}_{L}$ and $\tilde{t}_{L}$ parts of the coupling, this branching ratio cannot exceed $3 \%$ if only $\delta_{u}^{L R}$ is non-zero. If both parameters are non-zero, the branching fraction can be as large as about $4 \%$. Therefore, while the decays $\tilde{u}_{2} \rightarrow \tilde{u}_{1} Z^{0}$ and $\tilde{u}_{6} \rightarrow \tilde{u}_{2} Z^{0}$ are sizable over most of the parameter space considered, the other two are small and large statistics will be necessary to measure them.

We observe a similar picture in the context of decays into Higgs-bosons. Again, the branching fractions are generally smaller than for the Z-boson due to scalar phase space. The decay $\tilde{u}_{2} \rightarrow \tilde{u}_{1} h^{0}$ can only open for non-zero values of $\delta_{u}^{R R}$, while the branching ratio of $\tilde{u}_{2} \rightarrow \tilde{u}_{1} h^{0}$ decreases for $\left|\delta_{u}^{R R}\right| \rightarrow 1$ and increases for $\left|\delta_{u}^{L R}\right| \rightarrow 1$. For kinematical reasons, the decay of $\tilde{u}_{6}$ into $\tilde{u}_{2}$ is only allowed for large $\left|\delta_{u}^{R R}\right| \gtrsim 0.5$. In contrast to the variation of $\delta_{u}^{R R}$ only, the branching fraction $\operatorname{BR}\left(\tilde{u}_{4} \rightarrow \tilde{u}_{1} h^{0}\right)$ can here reach values of $7 \%$. This shows again, that combinations of NMFV-parameters can emphasise certain signatures.

Looking at decays into W-bosons, the channel $\tilde{d}_{5} \rightarrow \tilde{u}_{1} W^{-}$can open beside the two original (see table 3) ones, $\tilde{d}_{1} \rightarrow \tilde{u}_{1} W^{-}$and $\tilde{d}_{2} \rightarrow \tilde{u}_{1} W^{-}$. The branching fraction of 

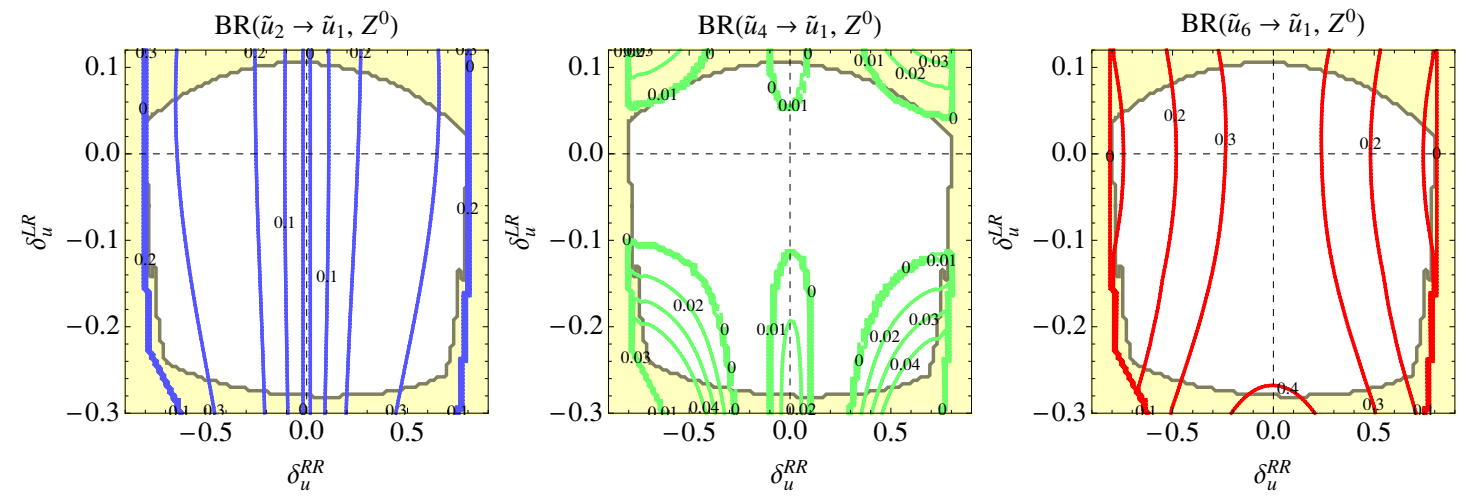

Figure 18. Dependence of the branching ratios of selected up-type squarks into Z-bosons on the NMFV-parameters $\delta_{u}^{R R}$ and $\delta_{u}^{L R}$ around the reference scenario SPS1a'. The shaded region indicates the experimentally disfavoured points.
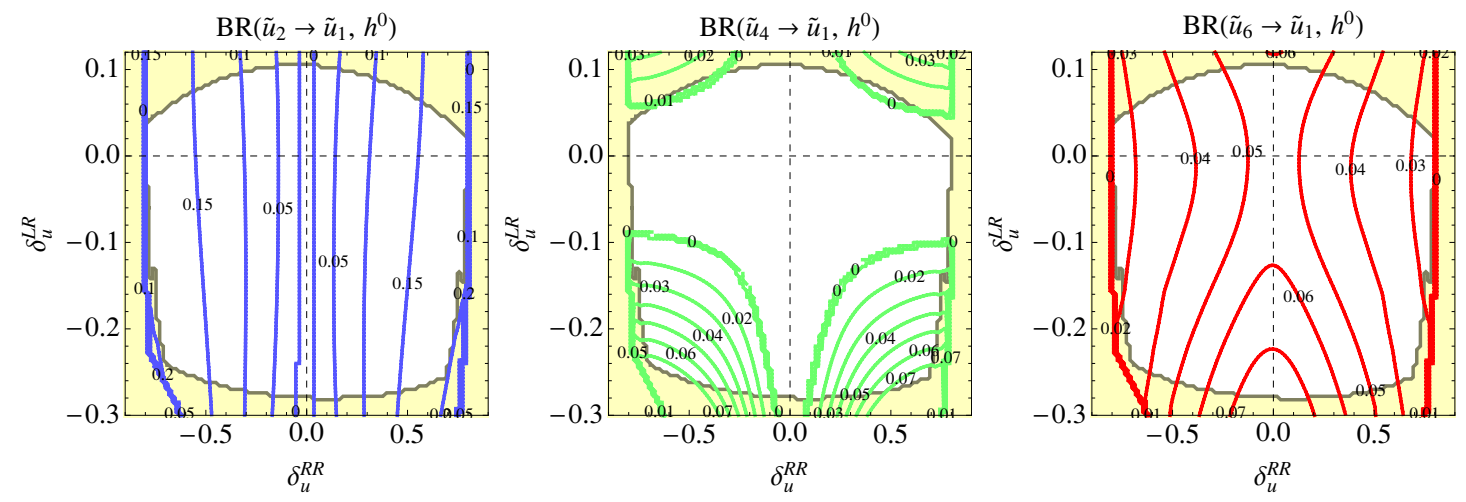

Figure 19. Dependence of the branching ratios of selected up-type squarks into Higgs-bosons on the NMFV-parameters $\delta_{u}^{R R}$ and $\delta_{u}^{L R}$ around the reference scenario SPS1a'. The shaded region indicates the experimentally disfavoured points.

the additional channel reaches about $20 \%$ within the experimentally allowed region of the NMFV-parameters. This is possible due to the loop-induced $\tilde{s}_{L}-\tilde{b}_{L}$ mixing. The branching fractions depend therefore only very weakly on the parameter $\delta_{u}^{R R}$, as these are left-left transitions and the impact is only via the phase space, and thus are not shown separately as the main information is already contained in figure 14 .

Last but not least, we mention that in case of SPS1b regions in parameter space are found where $\tilde{u}_{6}$ decays into three different final state squarks $\tilde{d}_{1,2,3}$ and a $W^{+}$. This becomes possible only if both NMFV-parameters $\delta_{u}^{R R}$ and $\delta_{u}^{L R}$ are non-zero.

In order to give a compact overview over the various signals, we summarise selected parameter configurations for the two reference scenarios and related NMFV-signatures in table 6 .

\section{Conclusion}

In summary, we have discussed which signatures related to bosonic decays of squarks could be able to challenge the hypothesis of minimal flavour violation in supersymmetry. There- 


\begin{tabular}{|c|c|c|c|}
\hline \multicolumn{4}{|c|}{ SPS1a' } \\
\hline \multicolumn{2}{|c|}{$\delta^{L L}=-0.09, \delta_{u}^{L R}=0.24$} & \multicolumn{2}{|c|}{$\delta_{u}^{R R}=0.648, \delta_{u}^{L R}=-0.24$} \\
\hline & & $\operatorname{BR}\left(\tilde{u}_{2} \rightarrow \tilde{u}_{1} Z^{0}\right)$ & $33 \%$ \\
\hline $\operatorname{BR}\left(\tilde{d}_{1} \rightarrow \tilde{u}_{1} W^{-}\right)$ & $=24 \%$ & $\operatorname{BR}\left(\tilde{u}_{4} \rightarrow \tilde{u}_{1} Z^{0}\right)$ & $19 \%$ \\
\hline $\operatorname{BR}\left(\tilde{d}_{2} \rightarrow \tilde{u}_{1} W^{-}\right)$ & $=14 \%$ & $\operatorname{BR}\left(\tilde{u}_{6} \rightarrow \tilde{u}_{1} Z^{0}\right)$ & $19 \%$ \\
\hline $\operatorname{BR}\left(\tilde{d}_{5} \rightarrow \tilde{u}_{1} W^{-}\right)$ & $=10 \%$ & $\operatorname{BR}\left(\tilde{u}_{6} \rightarrow \tilde{u}_{2} Z^{0}\right)$ & $1.7 \%$ \\
\hline $\operatorname{BR}\left(\tilde{d}_{6} \rightarrow \tilde{u}_{1} W^{-}\right)$ & $=30 \%$ & $\operatorname{BR}\left(\tilde{u}_{2} \rightarrow \tilde{u}_{1} h^{0}\right)$ & $19 \%$ \\
\hline $\operatorname{BR}\left(\tilde{u}_{2} \rightarrow \tilde{u}_{1} Z^{0}\right)$ & $2.5 \%$ & $\operatorname{BR}\left(\tilde{u}_{4} \rightarrow \tilde{u}_{1} h^{0}\right)$ & $6.7 \%$ \\
\hline $\operatorname{BR}\left(\tilde{u}_{6} \rightarrow \tilde{u}_{1} Z^{0}\right)$ & $34 \%$ & $\operatorname{BR}\left(\tilde{u}_{6} \rightarrow \tilde{u}_{1} h^{0}\right)$ & $3.7 \%$ \\
\hline & & $\operatorname{BR}\left(\tilde{u}_{6} \rightarrow \tilde{u}_{2} h^{0}\right)$ & $4.3 \%$ \\
\hline
\end{tabular}

\begin{tabular}{|c|c|c|}
\hline \multicolumn{4}{|c|}{$\mathrm{SPS} 1 \mathrm{~b}$} \\
\hline$\delta^{L L}=0.036, \delta_{u}^{L R}=-0.099$ & $\delta_{u}^{R R}=0.798, \delta_{u}^{L R}=-0.21$ \\
$\operatorname{BR}\left(\tilde{d}_{1} \rightarrow \tilde{u}_{1} W^{-}\right)=5.7 \%$ & $\operatorname{BR}\left(\tilde{u}_{2} \rightarrow \tilde{u}_{1} Z^{0}\right)=21 \%$ \\
$\operatorname{BR}\left(\tilde{u}_{2} \rightarrow \tilde{u}_{1} W^{-}\right)=13 \%$ & $\operatorname{BR}\left(\tilde{u}_{6} \rightarrow \tilde{u}_{1} Z^{0}\right)=5.2 \%$ \\
$\operatorname{BR}\left(\tilde{d}_{5} \rightarrow \tilde{u}_{1} W^{-}\right)=5.3 \%$ & $\operatorname{BR}\left(\tilde{u}_{6} \rightarrow \tilde{u}_{2} Z^{0}\right)=3.4 \%$ \\
$\operatorname{BR}\left(\tilde{d}_{6} \rightarrow \tilde{u}_{1} W^{-}\right)=7.1 \%$ & $\operatorname{BR}\left(\tilde{u}_{6} \rightarrow \tilde{u}_{4} Z^{0}\right)=1.6 \%$ \\
$\operatorname{BR}\left(\tilde{u}_{2} \rightarrow \tilde{u}_{1} Z^{0}\right)=5.8 \%$ & $\operatorname{BR}\left(\tilde{u}_{2} \rightarrow \tilde{u}_{1} h^{0}\right)=16 \%$ \\
$\operatorname{BR}\left(\tilde{u}_{6} \rightarrow \tilde{u}_{1} Z^{0}\right)=12 \%$ & $\operatorname{BR}\left(\tilde{u}_{4} \rightarrow \tilde{u}_{1} h^{0}\right)=19 \%$ \\
$\operatorname{BR}\left(\tilde{u}_{2} \rightarrow \tilde{u}_{1} h^{0}\right)=1.1 \%$ & $\operatorname{BR}\left(\tilde{u}_{6} \rightarrow \tilde{u}_{1} h^{0}\right)=1.4 \%$ \\
$\operatorname{BR}\left(\tilde{u}_{6} \rightarrow \tilde{u}_{1} h^{0}\right)=5.7 \%$ & $\operatorname{BR}\left(\tilde{u}_{6} \rightarrow \tilde{u}_{2} h^{0}\right)=4.8 \%$ \\
& $\operatorname{BR}\left(\tilde{u}_{6} \rightarrow \tilde{u}_{4} h^{0}\right)=2.0 \%$ \\
\hline
\end{tabular}

Table 6. Branching ratios of squark decays leading to NMFV-signatures for selected parameter points with two non-zero NMFV-parameters.

fore, we have investigated in detail the effect of non-minimally flavour-violating couplings of squarks to gauge- and Higgs-bosons within the MSSM. Starting from the benchmark scenarios SPS1a' and SPS1b we have introduced off-diagonal, i.e. flavour violating, parameters in the mass matrices of up- and down-type squarks.

After discussing their contributions to squark self-energy at the tree- or the one-loop level, we have studied in detail their implications for the decay of squarks into gauge- and Higgs-bosons. In particular, we have shown that each of the parameters inducing a flavour mixing between second and third generation squarks has a special characteristic, which is independent of the exact reference scenario. Combination of two parameters leads to a superposition of the associated effects on the mass spectrum and the decay signatures.

Typical signatures of non-minimally flavour-violating couplings can be observed in wide ranges of the analyzed parameter space: they involve either multiple decay modes of one single squark state into final states with gauge or Higgs bosons. The second possibility is that more than one squarks decay into the same final state containing a squark and a 
Z/Higgs-boson or that more than two squarks decay into the same final state containing a squark and W-boson or the charged Higgs boson. Clearly disentangling these final states is experimentally challenging and further detailed Monte Carlo studies will be necessary to demonstrate the feasibility of this idea.

We note that the detailed results presented here depend on the SUSY point chosen, e.g., the dominance of final states containing $W$-bosons ( $Z$ - and neutral Higgs bosons) in case of down-type squarks (up-type) squarks changes as sign of NMFV. Other SUSY breaking schemes and/or additional $D$-term contributions stemming from the breaking of larger rank groups to the SM gauge group might change the hierarchy between the soft SUSY breaking parameters leading to additional decay modes and/or suppressing the modes discussed here due to different kinematics. However, in general one gets sizable NMFV decay branching ratios except for the regions where all squark masses parameters squared are nearly degenerate and at the same time all left-right mixing entries are small.

As final result, if it will be possible to observe squarks at the LHC and to reconstruct their decay channels, the observation of the discussed signal would exclude the hypothesis of minimal flavour violation. Then, the purely bosonic decay modes discussed in this paper would deliver complementary information w.r.t. fermionic decays into charginos, neutralinos, or gluinos, which will be helpful for reconstructing the couplings and mass parameters of supersymmetric partners of the Standard Model particles.

\section{Acknowledgments}

We thank T. Hurth for useful discussions and F. Staub for cross checking the formulae given in appendix B. This work has been supported by the DFG, project number PO-1337/1-1. B.H. acknowledges support from the Hamburg Excellence Cluster "Connecting particles to the cosmos" and W.P. from the Alexander von Humboldt Foundation and the Spanish grant FPA2008-00319/FPA.

\section{A Couplings}

In the following, we give a compilation of the couplings of squarks to vector- and Higgsbosons taking into account the most general squark mixing as described in section 2. All couplings are given in both the super-CKM basis, $\tilde{q}^{(\mathrm{s})}$, and the mass eigenbasis of the squarks, $\tilde{q}^{(\mathrm{m})}$.

\section{A.1 Squark-squark-vector couplings}

Since gluons and photons are gauge bosons of the unbroken symmetry $\mathrm{SU}(3) \times \mathrm{U}(1)_{\mathrm{em}}$ and couple in equal manner to left- and right-handed squarks, their couplings to the physical mass eigenstates are not influenced by the presence of non-minimally flavour-violating terms. The latter only affect the couplings to Z- and W-bosons. In the super-CKM basis 
and using the same notation as in section 2, the relevant terms are given by the Lagrangian

$$
\begin{aligned}
\mathcal{L}_{\tilde{q} \tilde{q}^{\prime} V}= & -i \frac{2 g_{2}}{\cos \theta_{W}} Z_{\mu}\left[\tilde{q}_{L i}^{*(s)}\left(T_{3}^{\tilde{q}}-e_{\tilde{q}} \sin ^{2} \theta_{W}\right) \overleftrightarrow{\partial^{\mu}} \tilde{q}_{L i}^{(s)}-\tilde{q}_{R i}^{*(s)} e_{\tilde{q}} \sin ^{2} \theta_{W} \overleftrightarrow{\partial^{\mu}} \tilde{q}_{R i}^{(s)}\right] \\
& -i \sqrt{2} g_{2}\left[W_{\mu}^{+} \tilde{u}_{L i}^{*(s)}\left(V_{\mathrm{CKM}}\right)_{i j} \overleftrightarrow{\partial^{\mu}} \tilde{d}_{L j}^{(s)}+W_{\mu}^{-} \tilde{d}_{L i}^{*(s)}\left(V_{\mathrm{CKM}}^{\dagger}\right)_{i j} \overleftrightarrow{\partial^{\mu}} \tilde{u}_{L j}^{(s)}\right] \\
& -2 i e A_{\mu}\left[\tilde{q}_{L i}^{*(s)} e_{\tilde{q}} \overleftrightarrow{\partial^{\mu}} \tilde{q}_{L i}^{(s)}+\tilde{q}_{R i}^{*(s)} e_{\tilde{q}} \overleftrightarrow{\partial^{\mu}} \tilde{q}_{R i}^{(s)}\right]
\end{aligned}
$$

These terms are almost identical to the quark-quark-vector couplings with the difference that the squark fields have to be transformed to the mass eigenbasis $\tilde{q}^{(\mathrm{m})}$ according to

$$
\tilde{q}_{L i}^{(s)}=\sum_{t=1}^{6}\left(\mathcal{R}_{\tilde{q}}^{\dagger}\right)_{i t} \tilde{q}_{t}^{(\mathrm{m})}, \quad \tilde{q}_{R i}^{(s)}=\sum_{t=1}^{6}\left(\mathcal{R}_{\tilde{q}}^{\dagger}\right)_{(i+3) t} \tilde{q}_{t}^{(\mathrm{m})} .
$$

The resulting coupling terms in the mass basis are given by

$$
\begin{aligned}
\mathcal{L}_{\tilde{q} \tilde{q}^{\prime} V}= & -2 i \frac{g_{2}}{\cos \theta_{W}}\left[T_{3}^{\tilde{q}}\left(\mathcal{R}_{\tilde{q}}\right)_{s i}\left(\mathcal{R}_{\tilde{q}}^{\dagger}\right)_{i t}-e_{\tilde{q}} \sin ^{2} \theta_{W} \delta_{s t}\right] Z_{\mu} \tilde{q}_{s}^{*(\mathrm{~m})} \overleftrightarrow{\partial^{\mu}} \tilde{q}_{t}^{(\mathrm{m})} \\
& -2 i e e_{\tilde{q}} A_{\mu} \tilde{q}_{s}^{*(m)} \overleftrightarrow{\partial^{\mu}} \tilde{q}_{t}^{(\mathrm{m})} \delta_{s t}-2 i \frac{g_{2}}{\sqrt{2}}\left(\mathcal{R}_{\tilde{u}}\right)_{s i}\left(V_{\mathrm{CKM}}\right)_{i j}\left(\mathcal{R}_{\tilde{d}}^{\dagger}\right)_{j t} W_{\mu}^{+} \tilde{u}_{s}^{*(m)} \overleftrightarrow{\partial}^{\mu} \tilde{d}_{t}^{(\mathrm{m})} \\
& -2 i \frac{g_{2}}{\sqrt{2}}\left(\mathcal{R}_{\tilde{d}}\right)_{s i}\left(V_{\mathrm{CKM}}^{\dagger}\right)_{i j}\left(\mathcal{R}_{\tilde{u}}^{\dagger}\right)_{j t} W_{\mu}^{-} \tilde{d}_{s}^{*(m)} \stackrel{\leftrightarrow}{\partial^{\mu}} \tilde{u}_{t}^{(\mathrm{m})}
\end{aligned}
$$

Here, in addition to the CKM-matrix, the rotation matrices $\mathcal{R}_{\tilde{q}}$ enter the couplings explicitly, leading to NMFV-effects, e.g., in squark decays. While for couplings with W-bosons, both rotation matrices are present, in couplings to Z-bosons, only products of two entries of the same rotation matrix appear. In consequence, the couplings to $\mathrm{W}$-bosons are affected by all NMFV-parameters defined in section 2, while for a given squark decay into a Z-boson only at most four of them are relevant. Note that, although the matrices $\mathcal{R}_{\tilde{q}}$ are unitary, the product $\left(\mathcal{R}_{\tilde{q}}\right)_{s i}\left(\mathcal{R}_{\tilde{q}}^{\dagger}\right)_{i t} \neq \delta_{s t}$, since no summation over the right-handed indices is performed. The term including the photon field $A_{\mu}$ is included only for completeness.

\section{A.2 Squark-squark-Higgs couplings}

In the super-CKM basis, the Lagrangian including the coupling of squarks to the lighter neutral Higgs boson, $h^{0}$, is given by

$$
\begin{aligned}
\mathcal{L}_{\tilde{u} \tilde{u} h^{0}}= & -\frac{g_{2}}{2 m_{W}} h^{0}\left[\tilde{u}_{L i}^{*(s)} \tilde{u}_{L j}^{(\mathrm{s})}\left(m_{W}^{2} \sin (\alpha+\beta)\left(1-\frac{1}{3} \tan ^{2} \theta_{W}\right) \delta_{i j}+2 \frac{\cos \alpha}{\sin \beta} m_{u, i}^{2} \delta_{i j}\right)\right. \\
& +\tilde{u}_{R i}^{*(s)} \tilde{u}_{R j}^{(\mathrm{s})}\left(+m_{W}^{2} \sin (\alpha+\beta) \frac{4}{3} \tan ^{2} \theta_{W} \delta_{i j}+2 \frac{\cos \alpha}{\sin \beta} m_{u, i}^{2} \delta_{i j}\right) \\
& \left.+\left[\tilde{u}_{R i}^{*(s)} \tilde{u}_{L j}^{(\mathrm{s})}\left(+\mu^{*} \frac{\sin \alpha}{\sin \beta} m_{u, i} \delta_{i j}+\frac{\cos \alpha}{\sin \beta} \frac{v_{u}}{\sqrt{2}}\left(\hat{T}_{U}\right)_{i j}\right)+\text { h.c. }\right]\right]
\end{aligned}
$$


and

$$
\begin{aligned}
\mathcal{L}_{\tilde{d} \tilde{d} h^{0}}= & \frac{g_{2}}{2 m_{W}} h^{0}\left[\tilde{d}_{L i}^{*(s)} \tilde{d}_{L j}^{(\mathrm{s})}\left(m_{W}^{2} \sin (\alpha+\beta)\left(1+\frac{1}{3} \tan ^{2} \theta_{W}\right) \delta_{i j}+2 \frac{\sin \alpha}{\cos \beta} m_{d, i}^{2} \delta_{i j}\right)\right. \\
& +\tilde{d}_{i}^{(s)} \tilde{d}_{R j}^{(\mathrm{s})}\left(m_{W}^{2} \sin (\alpha+\beta) \frac{2}{3} \tan ^{2} \theta_{W} \delta_{i j}+2 \frac{\sin \alpha}{\cos \beta} m_{d, i}^{2} \delta_{i j}\right) \\
& \left.+\left[\tilde{d}_{R i}^{*(s)} \tilde{d}_{L j}^{(\mathrm{s})}\left(\mu^{*} \frac{\cos \alpha}{\cos \beta} m_{d, i} \delta_{i j}+\frac{\sin \alpha}{\cos \beta} \frac{v_{d}}{\sqrt{2}}\left(\hat{T}_{D}\right)_{i j}\right)+\text { h.c. }\right]\right]
\end{aligned}
$$

for up- and down-type squarks, respectively. The terms including $\tan ^{2} \theta_{W}$ stem from the D-terms of the scalar potential and are flavour-universal. Expressions with quark masses $m_{u, d}$ are the Yukawa- and F-terms, and the trilinear couplings are explicit breaking terms that couple left-handed to right-handed squarks. Transformation into the mass basis of the squark fields, the above expressions become

$$
\begin{aligned}
\mathcal{L}_{\tilde{u} \tilde{u} h^{0}}= & -\frac{g_{2}}{2 m_{W}} \tilde{u}_{s}^{*(m)} \tilde{u}_{t}^{(\mathrm{m})} h^{0}\left[m _ { W } ^ { 2 } \operatorname { s i n } ( \alpha + \beta ) \left[\left(1-\frac{1}{3} \tan ^{2} \theta_{W}\right)\left(\mathcal{R}_{\tilde{u}}\right)_{s i}\left(\mathcal{R}_{\tilde{u}}^{\dagger}\right)_{i t}\right.\right. \\
& \left.+\frac{4}{3} \tan ^{2} \theta_{W}\left(\mathcal{R}_{\tilde{u}}\right)_{s(i+3)}\left(\mathcal{R}_{\tilde{u}}^{\dagger}\right)_{(i+3) t}\right] \\
& +2 \frac{\cos \alpha}{\sin \beta}\left[\left(\mathcal{R}_{\tilde{u}}\right)_{s i} m_{u, i}^{2}\left(\mathcal{R}_{\tilde{u}}^{\dagger}\right)_{i t}+\left(\mathcal{R}_{\tilde{u}}\right)_{s(i+3)} m_{u, i}^{2}\left(\mathcal{R}_{\tilde{u}}^{\dagger}\right)_{i+3 t}\right] \\
& +\frac{\sin \alpha}{\sin \beta}\left[\mu^{*}\left(\mathcal{R}_{\tilde{u}}\right)_{s(i+3)} m_{u, i}\left(\mathcal{R}_{\tilde{u}}^{\dagger}\right)_{i t}+\mu\left(\mathcal{R}_{\tilde{u}}\right)_{s i} m_{u, i}\left(\mathcal{R}_{\tilde{u}}^{\dagger}\right)_{(i+3) t}\right] \\
& \left.+\frac{\cos \alpha}{\sin \beta} \frac{v_{u}}{\sqrt{2}}\left[\left(\mathcal{R}_{\tilde{u}}\right)_{s(i+3)}\left(\hat{T}_{U}\right)_{i j}\left(\mathcal{R}_{\tilde{u}}^{\dagger}\right)_{j t}+\left(\mathcal{R}_{\tilde{u}}\right)_{s i}\left(\hat{T}_{U}^{\dagger}\right)_{i j}\left(\mathcal{R}_{\tilde{u}}^{\dagger}\right)_{(j+3) t}\right]\right]
\end{aligned}
$$

and

$$
\begin{aligned}
\mathcal{L}_{\tilde{d} \tilde{d} h^{0}}= & \frac{g_{2}}{2 m_{W}} \tilde{d}^{*(m)} \tilde{d}_{t}^{(\mathrm{m})} h^{0}\left[m _ { W } ^ { 2 } \operatorname { s i n } ( \alpha + \beta ) \left[\left(1+\frac{1}{3} \tan ^{2} \theta_{W}\right)\left(\mathcal{R}_{\tilde{d}}\right)_{s i}\left(\mathcal{R}_{\tilde{d}}^{\dagger}\right)_{i t}\right.\right. \\
& \left.+\frac{2}{3} \tan ^{2} \theta_{W}\left(\mathcal{R}_{\tilde{d}}\right)_{s(i+3)}\left(\mathcal{R}_{\tilde{d}}^{\dagger}\right)_{(i+3) t}\right] \\
& +2 \frac{\sin \alpha}{\cos \beta}\left[\left(\mathcal{R}_{\tilde{d}}\right)_{s i} m_{d, i}^{2}\left(\mathcal{R}_{\tilde{d}}^{\dagger}\right)_{i t}+\left(\mathcal{R}_{\tilde{d}}\right)_{s(i+3)} m_{d, i}^{2}\left(\mathcal{R}_{\tilde{d}}^{\dagger}\right)_{(i+3) t}\right] \\
& +\frac{\cos \alpha}{\cos \beta}\left[\mu^{*}\left(\mathcal{R}_{\tilde{d}}\right)_{s(i+3)} m_{d, i}\left(\mathcal{R}_{\tilde{d}}^{\dagger}\right)_{i t}+\mu\left(\mathcal{R}_{\tilde{d}}\right)_{s i} m_{d, i}\left(\mathcal{R}_{\tilde{d}}^{\dagger}\right)_{(i+3) t}\right] \\
& \left.+\frac{\sin \alpha}{\cos \beta} \frac{v_{d}}{\sqrt{2}}\left[\left(\mathcal{R}_{\tilde{d}}\right)_{s(i+3)}\left(\hat{T}_{D}\right)_{i j}\left(\mathcal{R}_{\tilde{d}}^{\dagger}\right)_{j t}+\left(\mathcal{R}_{\tilde{d}}\right)_{s i}\left(\hat{T}_{D}^{\dagger}\right)_{i j}\left(\mathcal{R}_{\tilde{d}}^{\dagger}\right)_{(j+3) t}\right]\right] .
\end{aligned}
$$

The couplings of squarks to the heavier neutral Higgs-boson $H^{0}$ are obtained by replacing $h^{0} \rightarrow H^{0}, \sin \alpha \rightarrow \cos \alpha$, and $\cos \alpha \rightarrow-\sin \alpha$.

The pseudoscalar Higgs-boson $A^{0}$ is a mixture of the imaginary parts of the neutral components of the two doublets. Therefore it is anti-hermitian and the real diagonal contributions to the couplings vanish. The structure of the remaining terms is rather simple, the corresponding Lagrangian in the super-CKM basis is given by

$$
\begin{aligned}
\mathcal{L}_{\tilde{q} \tilde{q} A^{0}}= & -i \frac{g_{2}}{2 m_{W}} A^{0}\left[\tilde{d}_{R i}^{*(s)} \tilde{d}_{L j}^{(\mathrm{s})}\left(\mu^{*} m_{d, i} \delta_{i j}+\tan \beta \frac{v_{d}}{\sqrt{2}}\left(\hat{T}_{D}\right)_{i j}\right)\right. \\
& \left.+\tilde{u}_{R i}^{*(s)} \tilde{u}_{L j}^{(\mathrm{s})}\left(\mu^{*} m_{u, i} \delta_{i j}+\cot \beta \frac{v_{u}}{\sqrt{2}}\left(\hat{T}_{U}\right)_{i j}\right)+\text { h.c. }\right] .
\end{aligned}
$$


Transformation into the mass eigenbasis leads to

$$
\begin{aligned}
\mathcal{L}_{\tilde{d} \tilde{d} A^{0}}= & -i \frac{g_{2}}{2 m_{W}} \tilde{d}_{s}^{*(m)} \tilde{d}_{t}^{(\mathrm{m})} A^{0}\left[\mu^{*}\left(\mathcal{R}_{\tilde{d}}\right)_{s(i+3)} m_{d, i}\left(\mathcal{R}_{\tilde{d}}^{\dagger}\right)_{i t}\right. \\
& \left.+\tan \beta \frac{v_{d}}{\sqrt{2}}\left(\mathcal{R}_{\tilde{d}}\right)_{s(i+3)}\left(\hat{T}_{D}\right)_{i j}\left(\mathcal{R}_{\tilde{d}}^{\dagger}\right)_{j t}+\text { h.c. }\right]
\end{aligned}
$$

and

$$
\begin{aligned}
\mathcal{L}_{\tilde{u} \tilde{u} A^{0}}= & -i \frac{g_{2}}{2 m_{W}} \tilde{u}_{s}^{*(\mathrm{~m})} \tilde{u}_{t}^{(\mathrm{m})} A^{0}\left[\mu^{*}\left(\mathcal{R}_{\tilde{u}}\right)_{s(i+3)} m_{u i}\left(\mathcal{R}_{\tilde{u}}^{\dagger}\right)_{i t}\right. \\
& \left.+\cot \beta \frac{v_{u}}{\sqrt{2}}\left(\mathcal{R}_{\tilde{u}}\right)_{s(i+3)}\left(\hat{T}_{U}\right)_{i j}\left(\mathcal{R}_{\tilde{u}}^{\dagger}\right)_{j t}+\text { h.c. }\right] .
\end{aligned}
$$

Finally, the couplings of charged Higgs-bosons to squarks are given in super-CKM basis by

$$
\begin{aligned}
\mathcal{L}_{\tilde{q} \tilde{q} H^{ \pm}}= & \frac{g_{2}}{\sqrt{2} m_{W}} H^{-}\left[\tilde { d } _ { L i } ^ { * ( s ) } \tilde { u } _ { L j } ^ { ( \mathrm { s } ) } \left(-m_{W}^{2} \sin 2 \beta\left(V_{\mathrm{CKM}}^{\dagger}\right)_{i j}\right.\right. \\
& \left.+\tan \beta m_{d, i}^{2}\left(V_{\mathrm{CKM}}^{\dagger}\right)_{i j}+\cot \beta\left(V_{\mathrm{CKM}}^{\dagger}\right)_{i j} m_{u, j}^{2}\right) \\
& +\tilde{d}_{R i}^{*(s)} \tilde{u}_{L j}^{(\mathrm{s})}\left(\mu^{*} m_{d, i} \delta_{i j}+\tan \beta \frac{v_{d}}{\sqrt{2}}\left(\hat{T}_{D}\right)_{i j}\right) \\
& +\tilde{d}_{L i}^{*(s)} \tilde{u}_{R j}^{(\mathrm{s})}\left(\mu m_{u, i} \delta_{i j}+\cot \beta \frac{v_{u}}{\sqrt{2}}\left(\hat{T}_{U}^{\dagger}\right)_{i j}\right) \\
& \left.+\tilde{d}_{R i}^{*(s)} \tilde{u}_{R j}^{(\mathrm{s})}(\tan \beta+\cot \beta) m_{d, i}\left(V_{\mathrm{CKM}}^{\dagger}\right)_{i j} m_{u j}\right]+ \text { h.c. },
\end{aligned}
$$

leading to the following expression in the squark mass basis

$$
\begin{aligned}
\mathcal{L}_{\tilde{q} \tilde{q} H^{ \pm}}= & \frac{g_{2}}{\sqrt{2} m_{W}} \tilde{d}_{s}^{*(m)} \tilde{u}_{t}^{(\mathrm{m})} H^{-}\left[-m_{W}^{2} \sin 2 \beta\left(\mathcal{R}_{\tilde{d}}\right)_{s i}\left(V_{\mathrm{CKM}}^{\dagger}\right)_{i j}\left(\mathcal{R}_{\tilde{u}}^{\dagger}\right)_{j t}\right. \\
& +\tan \beta\left(\mathcal{R}_{\tilde{d}}\right)_{s i} m_{d, i}^{2}\left(V_{\mathrm{CKM}}^{\dagger}\right)_{i j}\left(\mathcal{R}_{\tilde{u}}^{\dagger}\right)_{j t}+\mu^{*}\left(\mathcal{R}_{\tilde{d}}\right)_{s(i+3)} m_{d, i}\left(\mathcal{R}_{\tilde{u}}^{\dagger}\right)_{i t} \\
& +\tan \beta \frac{v_{d}}{\sqrt{2}}\left(\mathcal{R}_{\tilde{d}}\right)_{s(i+3)}\left(\hat{T}_{D}\right)_{i j}\left(\mathcal{R}_{\tilde{u}}^{\dagger}\right)_{j t} \\
& +\cot \beta\left(\mathcal{R}_{\tilde{d}}\right)_{s i}\left(V_{\mathrm{CKM}}^{\dagger}\right)_{i j} m_{u, j}^{2}\left(\mathcal{R}_{\tilde{u}}^{\dagger}\right)_{j t}+\mu\left(\mathcal{R}_{\tilde{d}}\right)_{s i} m_{u, i}\left(\mathcal{R}_{\tilde{u}}^{\dagger}\right)_{(i+3) t} \\
& +\cot \beta \frac{v_{u}}{\sqrt{2}}\left(\mathcal{R}_{\tilde{d}}\right)_{s i}\left(\hat{T}_{U}^{\dagger}\right)_{i j}\left(\mathcal{R}_{\tilde{u}}^{\dagger}\right)_{(j+3) t} \\
& \left.+(\tan \beta+\cot \beta)\left(\mathcal{R}_{\tilde{d}}\right)_{s(i+3)} m_{d, i}\left(V_{\mathrm{CKM}}^{\dagger}\right)_{i j} m_{u, j}\left(\mathcal{R}_{\tilde{u}}^{\dagger}\right)_{(j+3) t}\right]+ \text { h.c.. }
\end{aligned}
$$

\section{B One-loop mass matrices}

The one-loop mass matrizes are given by the equations below and have been cross-checked using the package SARAH [37, 38]. The mass eigenvalues squared are obtained by taking the real part of the poles of the propagator matrix

$$
\operatorname{Det}\left[p_{k}^{2}-\mathcal{M}_{\tilde{q}}^{2}\left(p_{k}^{2}\right)\right]=0, m_{\tilde{q}_{k}}^{2}=\operatorname{Re}\left(p_{k}^{2}\right), k=1, \ldots, 6
$$


where

$$
\left(\mathcal{M}_{\tilde{q}}^{2}\left(p_{k}^{2}\right)\right)_{i j}=\left(\mathcal{M}_{\tilde{q}}^{2}\right)_{i j}-\left(\Pi\left(p_{k}^{2}\right)\right)_{i j}
$$

In this expression, $\mathcal{M}_{\tilde{q}}^{2}$ is the tree-level mass matrix given in eq. (2.1) where all entries contain running $\overline{\mathrm{DR}}$ parameters at a common scale $Q$. $\Pi\left(Q^{2}\right)$ contains the squark selfenergy functions evaluated at the scale $Q$.

We obtain

$$
\begin{aligned}
& \left(\Pi\left(p^{2}\right)\right)_{i j}=\sum_{n=1}^{6}\left[\frac{4}{3} F_{0}\left(p^{2}, m_{\tilde{q}_{n}}^{2}, 0\right) \Gamma_{\tilde{\tilde{q}}_{j}, G, \tilde{q}_{n}}^{*} \Gamma_{\hat{\tilde{q}}_{i}, G, \tilde{q}_{n}}+F_{0}\left(p^{2}, m_{\tilde{q}_{n}}^{2}, 0\right) \Gamma_{\tilde{\tilde{q}}_{j}, \gamma, \tilde{q}_{n}}^{*} \Gamma_{\hat{\tilde{q}}_{i}, \gamma, \tilde{q}_{n}}\right. \\
& +F_{0}\left(p^{2}, m_{\tilde{q}_{n}^{\prime}}^{2}, m_{W}^{2}\right) \Gamma_{\tilde{\tilde{q}}_{j}, W^{+}, \tilde{q}_{n}}^{*} \Gamma_{\hat{\tilde{q}}_{i}, W^{+}, \tilde{q}_{n}^{\prime}}+F_{0}\left(p^{2}, m_{\tilde{q}_{n}}^{2}, m_{Z}^{2}\right) \Gamma_{\tilde{\tilde{q}}_{j}, Z, \tilde{q}_{n}}^{*} \Gamma_{\hat{\tilde{q}}_{i}, Z, \tilde{q}_{n}} \\
& +2 A_{0}\left(m_{Z}^{2}\right) \Gamma_{\hat{\tilde{q}}_{i}^{*}, \hat{\tilde{q}}_{j}, Z, Z}+4 A_{0}\left(m_{W}^{2}\right) \Gamma_{\hat{\tilde{q}}_{i}^{*}, \hat{\tilde{q}}_{j}, W^{+}, W^{-}} \\
& -\sum_{l=1}^{2}\left[\frac{1}{2} A_{0}\left(m_{h_{l}^{0}}^{2}\right) \Gamma_{\hat{\tilde{q}}_{i}^{*}, \hat{\tilde{q}}_{j}, h_{l}^{0}, h_{l}^{0}}+\frac{1}{2} A_{0}\left(m_{A_{l}^{0}}^{2}\right) \Gamma_{\hat{\tilde{q}}_{i}^{*}, \hat{\tilde{q}}_{j}, A_{l}^{0}, A_{l}^{0}}+A_{0}\left(m_{H_{l}^{+}}^{2}\right) \Gamma_{\hat{\tilde{q}}_{i}^{*}, \hat{\tilde{q}}_{j}, H_{l}^{-}, H_{l}^{+}}\right] \\
& +\sum_{l=1}^{2} \sum_{n=1}^{6}\left[B_{0}\left(p^{2}, m_{A_{l}^{0}}^{2}, m_{\tilde{q}_{n}}^{2}\right) \Gamma_{\hat{q}_{j}, A_{l}^{0}, \tilde{q}_{n}}^{*} \Gamma_{\hat{\tilde{q}}_{i}, A_{l}^{0}, \tilde{q}_{n}}+B_{0}\left(p^{2}, m_{h_{l}^{0}}^{2}, m_{\tilde{q}_{n}}^{2}\right) \Gamma_{\hat{q}_{j}, h_{l}^{0}, \tilde{q}_{n}}^{*} \Gamma_{\hat{\tilde{q}}_{i}, h_{l}^{0}, \tilde{q}_{n}}\right. \\
& \left.+B_{0}\left(p^{2}, m_{H_{l}^{+}}^{2}, m_{\tilde{u}_{n}}^{2}\right) \Gamma_{\tilde{\tilde{q}}_{j}, H_{l}^{+}, \tilde{q}_{n}^{\prime}}^{*} \Gamma_{\hat{\tilde{q}}_{i}, H_{l}^{+}, \tilde{q}_{n}^{\prime}}\right] \\
& -\sum_{l=1}^{6}\left[A_{0}\left(m_{\tilde{d}_{l}}^{2}\right) \Gamma_{\hat{\tilde{q}}_{i}^{*}, \hat{\tilde{q}}_{j}, \tilde{\tilde{d}}_{l}^{*}, \tilde{d}_{l}}+A_{0}\left(m_{\tilde{u}_{l}}^{2}\right) \Gamma_{\hat{\tilde{q}}_{i}^{*}, \hat{\tilde{q}}_{j}, \tilde{u}_{l}^{*}, \tilde{u}_{l}}+A_{0}\left(m_{\tilde{e}_{l}}^{2}\right) \Gamma_{\hat{\tilde{q}}_{i}^{*}, \hat{\tilde{q}}_{j}, \tilde{e}_{l}^{*}, \tilde{e}_{l}}\right] \\
& -\sum_{l=1}^{3} A_{0}\left(m_{\tilde{\nu}_{l}}^{2}\right) \Gamma_{\hat{\tilde{q}}_{i}^{*}, \hat{\tilde{q}}_{j}, \tilde{\nu}_{l}^{*}, \tilde{\nu}_{l}} \\
& +\frac{4}{3} \sum_{l=1}^{3}\left[G_{0}\left(p^{2}, m_{q_{l}}^{2}, m_{\tilde{g}}^{2}\right)\left(\Gamma_{\tilde{\tilde{q}}_{j}, q_{l}, \tilde{g}}^{L *} \Gamma_{\tilde{\tilde{q}}_{i}, q_{l}, \tilde{g}}^{L}+\Gamma_{\tilde{q}_{j}, q_{l}, \tilde{g}}^{* R} \Gamma_{\tilde{\tilde{q}}_{i}, q_{l}, \tilde{g}}^{R}\right)\right. \\
& \left.-2 m_{\tilde{g}} m_{q_{l}} B_{0}\left(p^{2}, m_{q_{l}}^{2}, m_{\tilde{g}}^{2}\right)\left(\Gamma_{\tilde{\tilde{q}}_{j}, q_{l}, \tilde{g}}^{L *} \Gamma_{\tilde{\tilde{q}}_{i}, q_{l}, \tilde{g}}^{R}+\Gamma_{\tilde{\tilde{q}}_{j}, q_{l}, \tilde{g}^{2}}^{R *} \Gamma_{\tilde{\tilde{q}}_{i}, q_{l}, \tilde{g}}^{L}\right)\right] \\
& +\sum_{l=1}^{2} \sum_{n=1}^{3}\left[G_{0}\left(p^{2}, m_{\tilde{\chi}_{l}^{+}}^{2}, m_{q_{n}^{\prime}}^{2}\right)\left(\Gamma_{\hat{\tilde{q}}_{j}, \tilde{\chi}_{l}^{+}, q_{n}^{\prime}}^{L *} \Gamma_{\hat{q}_{i}, \tilde{\chi}_{l}^{+}, q_{n}^{\prime}}^{L}+\Gamma_{\tilde{q}_{j}, \tilde{\chi}_{l}^{+}, q_{n}^{\prime}}^{R *} \Gamma_{\hat{\tilde{q}}_{i}, \tilde{\chi}_{l}^{+}, q_{n}^{\prime}}^{R}\right)\right. \\
& \left.-2 m_{\tilde{\chi}_{l}^{+}} m_{q_{n}^{\prime}} B_{0}\left(p^{2}, m_{\tilde{\chi}_{l}^{+}}^{2}, m_{q_{n}^{\prime}}^{2}\right)\left(\Gamma_{\hat{\tilde{q}}_{j}, \tilde{\chi}_{l}^{+}, q_{n}^{\prime}}^{L *} \Gamma_{\hat{\tilde{q}}_{i}, \tilde{\chi}_{l}^{+}, q_{n}^{\prime}}^{R}+\Gamma_{\hat{\tilde{q}}_{j}, \tilde{\chi}_{l}^{+}, q_{n}^{\prime}}^{R *} \Gamma_{\hat{q}_{i}, \tilde{\chi}_{l}^{+}, q_{n}^{\prime}}^{L}\right)\right] \\
& +\sum_{l=1}^{4} \sum_{n=1}^{3}\left[G_{0}\left(p^{2}, m_{\tilde{\chi}_{l}^{0}}^{2}, m_{q_{n}}^{2}\right)\left(\Gamma_{\tilde{\tilde{q}}_{j}, \tilde{\chi}_{l}^{0}, q_{n}}^{L *} \Gamma_{\tilde{\tilde{q}}_{i}, \tilde{\chi}_{l}^{0}, q_{n}}^{L}+\Gamma_{\tilde{\tilde{q}}_{j}, \tilde{\chi}_{l}^{0}, q_{n}}^{R *} \Gamma_{\tilde{\tilde{q}}_{i}, \tilde{\chi}_{l}^{0}, q_{n}}^{R}\right)\right. \\
& \left.-2 m_{\tilde{\chi}_{l}^{0}} m_{q_{n}} B_{0}\left(p^{2}, m_{\tilde{\chi}_{l}^{0}}^{2}, m_{q_{n}}^{2}\right)\left(\Gamma_{\hat{\tilde{q}}_{j}, \tilde{\chi}_{l}^{0}, q_{n}}^{L *} \Gamma_{\hat{\tilde{q}}_{i}, \tilde{\chi}_{l}^{0}, q_{n}}^{R}+\Gamma_{\hat{\tilde{q}}_{j}, \tilde{\chi}_{l}^{0}, q_{n}}^{R *} \Gamma_{\hat{\tilde{q}}_{i}, \tilde{\chi}_{l}^{0}, q_{n}}^{L}\right)\right],
\end{aligned}
$$

where the notation $\hat{\tilde{q}}_{i}$ indicates that the corresponding squark is in the electroweak eigen- 
basis. The following couplings are independent of the squark type:

$$
\begin{aligned}
\Gamma_{\hat{\tilde{q}}_{j}, g, \tilde{q}_{n}} & =g_{3}\left(\mathcal{R}_{\tilde{q}}\right)_{j n}, \\
\Gamma_{\tilde{\tilde{q}}_{j}, \gamma, \tilde{q}_{n}} & =e_{\tilde{q}} e\left(\mathcal{R}_{\tilde{q}}\right)_{j n} \\
\Gamma_{\hat{\tilde{q}}_{j}, Z, \tilde{q}_{n}} & =\frac{g_{2}}{\cos \theta_{W}}\left[T_{3}^{\tilde{q}} \Theta(4-j)-e_{\tilde{q}} \sin ^{2} \theta_{W}\right]\left(\mathcal{R}_{\tilde{q}}\right)_{j n}, \\
\Gamma_{\hat{\tilde{q}}_{i}^{*}, \hat{\tilde{q}}_{j}, Z, Z} & =\frac{2 g_{2}^{2}}{\cos ^{2} \theta_{W}}\left(T_{3}^{\tilde{q}} \Theta(4-i)-e_{q} \sin ^{2} \theta_{W}\right)^{2} \delta_{i j}, \\
\Gamma_{\hat{\tilde{q}}_{i}^{*}, \hat{q}_{j}, W^{+}, W^{-}} & =\frac{g_{2}^{2}}{2} \delta_{i j} \Theta(4-i) \\
\Gamma_{\hat{\tilde{q}}_{i}^{*}, \hat{\tilde{q}}_{j}, H_{l}^{-}, H_{l}^{+}} & =\left[\left(D_{\tilde{q}}^{\prime}-Y_{d, i}^{2} \Theta(4-i)\right)\left(R_{l 1}^{H^{+}}\right)^{2}-\left(D_{\tilde{q}}^{\prime}+Y_{u, i-3}^{2} \Theta(i-3)\right)\left(R_{l 2}^{H^{+}}\right)^{2}\right] \delta_{i j}, \\
\Gamma_{\hat{\tilde{q}}_{i}^{*}, \hat{\tilde{q}}_{j}, \tilde{\nu}_{l}^{*}, \tilde{\nu}_{l}} & =-2 D_{\tilde{q}} \delta_{i j}, \\
\Gamma_{\tilde{\tilde{q}}_{j}, q_{l}, \tilde{g}^{\prime}}^{L} & =\sqrt{2} g_{3} \delta_{i, l} e^{i \varphi_{\tilde{3}}} \\
\Gamma_{\hat{\tilde{q}}_{i}, q_{l}, \tilde{g}^{\prime}}^{R} & =\sqrt{2} g_{3} \delta_{i, l+3} e^{-i \varphi_{\tilde{3}}}
\end{aligned}
$$

For the down-squarks we have

$$
\begin{aligned}
& \Gamma_{\hat{\tilde{d}}_{j}, W^{+}, \tilde{u}_{n}}=\frac{g_{2}}{\sqrt{2}} \delta_{j k}\left(V_{\mathrm{CKM}}^{\dagger}\right)_{k l}\left(\mathcal{R}_{\tilde{u}}^{\dagger}\right)_{l n}, \\
& \Gamma_{\hat{\tilde{d}}_{i}, h_{l}^{0}, \tilde{d}_{n}}=\frac{g_{2}}{2 m_{W}}\left[m _ { W } ^ { 2 } ( R _ { l 2 } ^ { h ^ { 0 } } \operatorname { s i n } \beta - R _ { l 1 } ^ { h ^ { 0 } } \operatorname { c o s } \beta ) \left[\left(1+\frac{1}{3} \tan ^{2} \theta_{W}\right)\left(\mathcal{R}_{\tilde{d}}^{\dagger}\right)_{i n} \Theta(4-i)\right.\right. \\
& \left.+\frac{2}{3} \tan ^{2} \theta_{W}\left(\mathcal{R}_{\tilde{d}}^{\dagger}\right)_{i n} \Theta(i-3)\right] \\
& -2 \frac{R_{l 1}^{h^{0}}}{\cos \beta}\left[\left(\mathcal{R}_{\tilde{d}}^{\dagger}\right)_{i n} m_{d, i}^{2} \Theta(4-i)+\left(\mathcal{R}_{\tilde{d}}^{\dagger}\right)_{i n} m_{d, i-3}^{2} \Theta(i-3)\right] \\
& +\frac{R_{l 2}^{h^{0}}}{\cos \beta}\left[\mu^{*}\left(\mathcal{R}_{\tilde{d}}^{\dagger}\right)_{i n} m_{d, i} \Theta(4-i)+\mu\left(\mathcal{R}_{\tilde{d}}^{\dagger}\right)_{i n} m_{d, i-3} \Theta(i-3)\right] \\
& \left.-\frac{R_{l 1}^{h^{0}}}{\cos \beta} \frac{v_{d}}{\sqrt{2}}\left[\left(\mathcal{R}_{\tilde{d}}^{\dagger}\right)_{j n}\left(\hat{T}_{D}\right)_{j i} \Theta(4-i)+\left(\mathcal{R}_{\tilde{d}}^{\dagger}\right)_{j n}\left(\hat{T}_{D}^{\dagger}\right)_{j(i-3)} \Theta(i-3)\right]\right], \\
& \Gamma_{\hat{\tilde{d}}_{i}, A_{l}^{0}, \tilde{d}_{n}}=\frac{R_{l 2}^{A^{0}}}{\sqrt{2}} \mu^{*}\left(\mathcal{R}_{\tilde{d}}^{\dagger}\right)_{i n} Y_{d, i} \Theta(4-i)-R_{l 1}^{A^{0}}\left(\mathcal{R}_{\tilde{d}}^{\dagger}\right)_{j n}\left(\hat{T}_{D}\right)_{j(i-3)} \Theta(i-3), \\
& \Gamma_{\hat{\tilde{d}}_{i}, H_{l}^{+}, \tilde{u}_{n}}=\frac{g_{2}}{\sqrt{2} m_{W}}\left[-\frac{g_{2}}{\sqrt{2}} m_{W}^{2} \sin 2 \beta \Theta(4-i)\left(V_{\mathrm{CKM}}^{\dagger}\right)_{i j}\left(\mathcal{R}_{\tilde{u}}^{\dagger}\right)_{j n}\right. \\
& +\tan \beta \Theta(4-i) m_{d, i}^{2}\left(V_{\text {CKM }}^{\dagger}\right)_{i j}\left(\mathcal{R}_{\tilde{u}}^{\dagger}\right)_{j n}+\mu^{*} \Theta(i-3) m_{d, i}\left(\mathcal{R}_{\tilde{u}}^{\dagger}\right)_{i n} \\
& +\tan \beta \frac{v_{d}}{\sqrt{2}} \Theta(i-3)\left(\hat{T}_{D}\right)_{i j}\left(\mathcal{R}_{\tilde{u}}^{\dagger}\right)_{j n} \\
& +\cot \beta \Theta(4-i)\left(V_{\text {CKM }}^{\dagger}\right)_{i j} m_{u, j}^{2}\left(\mathcal{R}_{\tilde{u}}^{\dagger}\right)_{j n}+\mu \Theta(4-i) m_{u, i}\left(\mathcal{R}_{\tilde{u}}^{\dagger}\right)_{(i+3) n} \\
& +\cot \beta \frac{v_{u}}{\sqrt{2}} \Theta(4-i)\left(\hat{T}_{U}^{\dagger}\right)_{i j}\left(\mathcal{R}_{\tilde{u}}^{\dagger}\right)_{(j+3) n} \\
& \left.+(\tan \beta+\cot \beta) \Theta(i-3) m_{d, i}\left(V_{\mathrm{CKM}}^{\dagger}\right)_{i j} m_{u, j}\left(\mathcal{R}_{\tilde{u}}^{\dagger}\right)_{(j+3) t}\right] \\
& \Gamma_{\hat{\tilde{d}}_{i}^{*}, \hat{\tilde{d}}_{j}, h_{l}^{0}, h_{l}^{0}}=\left[-\left(D_{\tilde{q}}+\frac{1}{2} Y_{d, i}^{2} \Theta(4-i)+\frac{1}{2} Y_{d, i-3}^{2} \Theta(i-3)\right)\left(R_{l 1}^{h^{0}}\right)^{2}+D_{\tilde{q}}\left(R_{l 2}^{h^{0}}\right)^{2}\right] \delta_{i j},
\end{aligned}
$$




$$
\begin{aligned}
& \Gamma_{\hat{\tilde{d}}_{i}^{*}, \hat{d}_{j}, A_{l}^{0}, A_{l}^{0}}=\left[-\left(D_{\tilde{q}}+\frac{1}{2} Y_{d, i}^{2} \Theta(4-i)+\frac{1}{2} Y_{d, i-3}^{2} \Theta(i-3)\right)\left(R_{l 1}^{A^{0}}\right)^{2}+D_{\tilde{q}}\left(R_{l 2}^{A^{0}}\right)^{2}\right] \delta_{i j}, \\
& \Gamma_{\hat{\tilde{d}}_{i}^{*}, \hat{\tilde{d}}_{j}, \tilde{e}_{l}^{*}, \tilde{e}_{l}}=-g_{2}^{2}\left[\Theta(4-i)\left(\frac{1}{4}-\frac{1}{12} \tan ^{2} \theta_{W}\right) \sum_{k=1}^{3}\left(\mathcal{R}_{\tilde{l}}^{\dagger}\right)_{l k}\left(\mathcal{R}_{\tilde{l}}\right)_{k l}\right. \\
& -\Theta(i-3) \frac{1}{6} \tan ^{2} \theta_{W} \sum_{k=1}^{3}\left(\left(\mathcal{R}_{\tilde{l}}^{\dagger}\right)_{l k}\left(\mathcal{R}_{\tilde{l}}\right)_{k l}-2\left(\mathcal{R}_{\tilde{l}}^{\dagger}\right)_{l(k+3)}\left(\mathcal{R}_{\tilde{l}}\right)_{(k+3) l}\right) \\
& \left.+\Theta(4-i) \frac{1}{6} \tan ^{2} \theta_{W} \sum_{k=4}^{6}\left(\mathcal{R}_{\tilde{l}}^{\dagger}\right)_{l k}\left(\mathcal{R}_{\tilde{l}}\right)_{k l}\right] \delta_{i j} \\
& -\left(\sum_{k=1}^{3}\left(\mathcal{R}_{\tilde{l}}^{\dagger}\right)_{l k} Y_{l, k}\left(\mathcal{R}_{\tilde{l}}\right)_{(k+3) l}\right) Y_{d, i} \delta_{i(j+3)}-\left(\sum_{k=1}^{3}\left(\mathcal{R}_{\tilde{l}}^{\dagger}\right)_{l k} Y_{l, k}\left(\mathcal{R}_{\tilde{l}}\right)_{(k+3) l}\right)^{*} Y_{d, i} \delta_{(i+3) j} \\
& \Gamma_{\hat{\tilde{d}}_{i}, \tilde{\chi}_{l}^{+}, u_{n}}^{L}=-g_{2}\left(V_{\mathrm{CKM}}^{\dagger}\right)_{i n} V_{l 1}+Y_{u, n}\left(V_{\mathrm{CKM}}^{\dagger}\right)_{n(i-3)} V_{l 2} \\
& \Gamma_{\hat{\tilde{d}}_{i}, \tilde{\chi}_{l}^{+}, u_{n}}^{R}=\left(V_{\mathrm{CKM}}^{\dagger}\right)_{n i} Y_{d, i} U_{l 2} \\
& \Gamma_{\hat{\tilde{d}}_{j}, \tilde{\chi}_{l}^{0}, d_{n}}^{L}=-\sqrt{2} g_{2}\left(-\frac{1}{2} N_{l 2}-\frac{1}{6} \tan \theta_{W} N_{l 1}\right) \delta_{i n}-Y_{d, n} N_{l 3} \delta_{i(n+3)} \\
& \Gamma_{\tilde{\tilde{d}}_{i}, \tilde{\chi}_{l}^{0}, d_{n}}^{R}=\sqrt{2} e_{\tilde{u}} g_{2} \tan \theta_{W} N_{l 1}^{*} \delta_{i(n+3)}-Y_{d, n} N_{l 3}^{*} \delta_{i n} \\
& \Gamma_{\hat{\tilde{d}}_{i}^{*}, \hat{\tilde{d}}_{j}, \tilde{d}_{l}^{*}, \tilde{d}_{l}}=\frac{4}{3} g_{3}^{2}\left(\mathcal{R}_{\tilde{d}}\right)_{l i}\left(\mathcal{R}_{\tilde{d}}^{\dagger}\right)_{j l}[\Theta(i-3) \Theta(j-3)+\Theta(4-i) \Theta(4-j) \\
& -\Theta(i-3) \Theta(4-j)-\Theta(4-i) \Theta(j-3)] \\
& -\frac{g_{2}^{2}}{36}\left[\left[\left(9+\tan ^{2} \theta_{W}\right)\left(\mathcal{R}_{\tilde{d}}\right)_{l i}\left(\mathcal{R}_{\tilde{d}}^{\dagger}\right)_{j l}+3 \sum_{k=1}^{3}\left(\left(9+\tan ^{2} \theta_{W}\right)\left(\mathcal{R}_{\tilde{d}}\right)_{l k}\left(\mathcal{R}_{\tilde{d}}^{\dagger}\right)_{k l}\right.\right.\right. \\
& \left.\left.+2 \tan ^{2} \theta_{W}\left(\mathcal{R}_{\tilde{d}}\right)_{l(k+3)}\left(\mathcal{R}_{\tilde{d}}^{\dagger}\right)_{(k+3) l}\right) \delta_{i j}\right] \Theta(4-i) \Theta(4-j) \\
& +2 \tan ^{2} \theta_{W}\left[\left(\mathcal{R}_{\tilde{u}}\right)_{l i}\left(\mathcal{R}_{\tilde{u}}^{\dagger}\right)_{j l}\right. \\
& \left.+3 \sum_{k=1}^{3}\left(\left(\mathcal{R}_{\tilde{d}}\right)_{l k}\left(\mathcal{R}_{\tilde{d}}^{\dagger}\right)_{k l}+2\left(\mathcal{R}_{\tilde{d}}\right)_{l(k+3)}\left(\mathcal{R}_{\tilde{d}}^{\dagger}\right)_{(k+3) l}\right) \delta_{i j}\right] \Theta(i-3) \Theta(j-3) \\
& \left.+2 \tan ^{2} \theta_{W}\left(\mathcal{R}_{\tilde{d}}\right)_{l i}\left(\mathcal{R}_{\tilde{d}}^{\dagger}\right)_{j l}(\Theta(4-i) \Theta(j-3)+\Theta(i-3) \Theta(4-j))\right] \\
& -3 Y_{d, i} \delta_{i(j-3)} \Theta(4-i) \Theta(j-3) \sum_{k=1}^{3} Y_{d, k}\left(\mathcal{R}_{\tilde{d}}\right)_{l k}\left(\mathcal{R}_{\tilde{d}}^{\dagger}\right)_{(k+3) l} \\
& -3 Y_{d, j} \delta_{(i-3) j} \Theta(4-j) \Theta(i-3) \sum_{k=1}^{3} Y_{d, k}\left(\mathcal{R}_{\tilde{d}}\right)_{l k}\left(\mathcal{R}_{\tilde{d}}^{\dagger}\right)_{(k+3) l} \\
& -Y_{u, i} Y_{d, j}\left(\mathcal{R}_{\tilde{d}}\right)_{l(i+3)}\left(\mathcal{R}_{\tilde{d}}^{\dagger}\right)_{(j+3) l} \Theta(4-j) \Theta(4-i) \\
& -Y_{d,(i-3)} Y_{d,(j-3)}\left(\mathcal{R}_{\tilde{d}}\right)_{l(i-3)}\left(\mathcal{R}_{\tilde{d}}^{\dagger}\right)_{(j-3) l} \Theta(j-3) \Theta(i-3)
\end{aligned}
$$




$$
\begin{aligned}
\Gamma_{\hat{\tilde{d}}_{i}^{*}, \hat{\tilde{d}}_{j}, \tilde{u}_{l}^{*}, \tilde{u}_{l}=} & -g_{2}^{2}\left[\left(\left(V_{\mathrm{CKM}}\right)_{i l}\left(V_{\mathrm{CKM}}^{\dagger}\right)_{l j} \Theta(4-l)-\frac{3}{4} \delta_{i j}+\frac{1}{12} \tan ^{2} \theta_{W} \delta_{i j}\right)\right. \\
& \times \Theta(4-j) \Theta(4-i) \sum_{k=1}^{3}\left(\mathcal{R}_{\tilde{u}}\right)_{l k}\left(\mathcal{R}_{\tilde{u}}^{\dagger}\right)_{k l} \\
& +\frac{1}{6} \tan ^{2} \theta_{W} \delta_{i j} \Theta(j-3) \Theta(i-3) \sum_{k=1}^{3}\left(\mathcal{R}_{\tilde{u}}\right)_{l k}\left(\mathcal{R}_{\tilde{u}}^{\dagger}\right)_{k l} \\
& -\frac{1}{3} \tan ^{2} \theta_{W} \delta_{i j} \Theta(4-j) \Theta(4-i) \sum_{k=4}^{6}\left(\mathcal{R}_{\tilde{u}}\right)_{l k}\left(\mathcal{R}_{\tilde{u}}^{\dagger}\right)_{k l} \\
& \left.-\frac{2}{3} \tan ^{2} \theta_{W} \delta_{i j} \Theta(j-3) \Theta(i-3) \sum_{k=4}^{6}\left(\mathcal{R}_{\tilde{u}}\right)_{l k}\left(\mathcal{R}_{\tilde{u}}^{\dagger}\right)_{k l}\right] \\
& -\sum_{k=1}^{3}\left(\left(\mathcal{R}_{\tilde{u}}^{\dagger}\right)_{l k} Y_{u, k}\left(\mathcal{R}_{\tilde{u}}\right)_{(k+3) l}\right) Y_{d, i} \delta_{i(j+3)}-\sum_{k=1}^{3}\left(\left(\mathcal{R}_{\tilde{u}}^{\dagger}\right)_{l k} Y_{u, k}\left(\mathcal{R}_{\tilde{u}}\right)_{(k+3) l}\right)^{*} Y_{d, i} \delta_{(i+3) j}
\end{aligned}
$$

whereas for the up-squarks we have

$$
\begin{aligned}
& \Gamma_{\hat{\tilde{u}}_{j}, W^{+}, \tilde{d}_{n}}=\frac{g_{2}}{\sqrt{2}} \delta_{j k}\left(V_{\mathrm{CKM}}\right)_{k l}\left(\mathcal{R}_{\tilde{d}}^{\dagger}\right)_{l n}, \\
& \Gamma_{\hat{\tilde{u}}_{i}^{*}, \hat{\tilde{u}}_{j}, h_{l}^{0}, h_{l}^{0}}=\left[-D_{\tilde{q}}\left(R_{l 1}^{h^{0}}\right)^{2}+\left(D_{\tilde{q}}-\frac{1}{2} Y_{u, i}^{2} \Theta(4-i)-\frac{1}{2} Y_{u, i-3}^{2} \Theta(i-3)\right)\left(R_{l 2}^{h^{0}}\right)^{2}\right] \delta_{i j}, \\
& \Gamma_{\hat{\tilde{u}}_{i}^{*}, \hat{u}_{j}, A_{l}^{0}, A_{l}^{0}}=\left[-D_{\tilde{q}}\left(R_{l 1}^{A^{0}}\right)^{2}+\left(D_{\tilde{q}}-\frac{1}{2} Y_{u, i}^{2} \Theta(4-i)-\frac{1}{2} Y_{u, i-3}^{2} \Theta(i-3)\right)\left(R_{l 2}^{A^{0}}\right)^{2}\right] \delta_{i j}, \\
& \Gamma_{\hat{\tilde{u}}_{i}, h_{l}^{0}, \tilde{u}_{n}}=-\frac{g_{2}}{2 m_{W}}\left[m _ { W } ^ { 2 } ( R _ { l 2 } ^ { h ^ { 0 } } \operatorname { s i n } \beta - R _ { l 1 } ^ { h ^ { 0 } } \operatorname { c o s } \beta ) \left[\left(1-\frac{1}{3} \tan ^{2} \theta_{W}\right)\left(\mathcal{R}_{\tilde{u}}^{\dagger}\right)_{i n} \Theta(4-i)\right.\right. \\
& \left.+\frac{4}{3} \tan ^{2} \theta_{W}\left(\mathcal{R}_{\tilde{u}}^{\dagger}\right)_{i n} \Theta(i-3)\right] \\
& +2 \frac{R_{l 2}^{h^{0}}}{\sin \beta}\left[\left(\mathcal{R}_{\tilde{u}}^{\dagger}\right)_{i n} m_{u, i}^{2} \Theta(4-i)+\left(\mathcal{R}_{\tilde{u}}^{\dagger}\right)_{i n} m_{u, i-3}^{2} \Theta(i-3)\right] \\
& -\frac{R_{l 1}^{h^{0}}}{\sin \beta}\left[\mu^{*}\left(\mathcal{R}_{\tilde{u}}^{\dagger}\right)_{i n} m_{u, i} \Theta(4-i)+\mu\left(\mathcal{R}_{\tilde{u}}^{\dagger}\right)_{i n} m_{u, i} \Theta(i-3)\right] \\
& \left.+\frac{R_{l 2}^{h^{0}}}{\sin \beta} \frac{v_{u}}{\sqrt{2}}\left[\left(\mathcal{R}_{\tilde{u}}^{\dagger}\right)_{j n}\left(\hat{T}_{U}\right)_{j i} \Theta(4-i)+\left(\mathcal{R}_{\tilde{u}}^{\dagger}\right)_{j n}\left(\hat{T}_{U}^{\dagger}\right)_{j(i-3)} \Theta(i-3)\right]\right] \\
& \Gamma_{\hat{\tilde{u}}_{i}, A_{l}^{0}, \tilde{u}_{n}}=-\frac{R_{l 1}^{A^{0}}}{\sqrt{2}} \mu^{*}\left(\mathcal{R}_{\tilde{u}}^{\dagger}\right)_{i n} Y_{u i} \Theta(4-i)+R_{l 2}^{A^{0}}\left(\mathcal{R}_{\tilde{u}}^{\dagger}\right)_{j n}\left(\hat{T}_{U}\right)_{j i} \Theta(i-3)
\end{aligned}
$$




$$
\begin{aligned}
& \Gamma_{\hat{\tilde{u}}_{i}, H_{l}^{+}, \tilde{d}_{n}}=\frac{g_{2}}{\sqrt{2} m_{W}}\left[-m_{W}^{2} \sin 2 \beta \Theta(4-i)\left(V_{\mathrm{CKM}}\right)_{i j}\left(\mathcal{R}_{\tilde{d}}^{\dagger}\right)_{j n}\right. \\
& +\tan \beta \Theta(4-i)\left(V_{\mathrm{CKM}}\right)_{i j} m_{d, j}^{2}\left(\mathcal{R}_{\tilde{d}}^{\dagger}\right)_{j n}+\mu \Theta(4-i) m_{d, i}\left(\mathcal{R}_{\tilde{d}}^{\dagger}\right)_{(i+3) n} \\
& +\tan \beta \frac{v_{d}}{\sqrt{2}} \Theta(4-i)\left(\hat{T}_{D}^{\dagger}\right)_{i j}\left(\mathcal{R}_{\tilde{d}}^{\dagger}\right)_{(j+3) n} \\
& +\cot \beta \Theta(4-i) m_{u, i}^{2}\left(V_{\mathrm{CKM}}\right)_{i j}\left(\mathcal{R}_{\tilde{d}}^{\dagger}\right)_{j n}+\mu^{*} \Theta(i-3) m_{u, i}\left(\mathcal{R}_{\tilde{d}}^{\dagger}\right)_{i n} \\
& +\cot \beta \frac{v_{u}}{\sqrt{2}} \Theta(i-3)\left(\hat{T}_{U}\right)_{i j}\left(\mathcal{R}_{\tilde{d}}^{\dagger}\right)_{j n} \\
& \left.+(\tan \beta+\cot \beta) \Theta(i-3) m_{u, i}\left(V_{\mathrm{CKM}}\right)_{i j} m_{d, j}\left(\mathcal{R}_{\tilde{d}}^{\dagger}\right)_{(j+3) t}\right] \\
& \Gamma_{\hat{\tilde{u}}_{i}^{*}, \hat{u}_{j}, \tilde{e}_{l}^{*}, \tilde{e}_{l}}=-g_{2}^{2}\left[-\Theta(4-i)\left(\frac{1}{12} \tan ^{2} \theta_{W}+\frac{1}{4}\right) \sum_{k=1}^{3}\left(\mathcal{R}_{\tilde{l}}^{\dagger}\right)_{l k}\left(\mathcal{R}_{\tilde{l}}\right)_{k l}\right. \\
& +\Theta(i-3) \frac{1}{3} \tan ^{2} \theta_{W} \sum_{k=1}^{3}\left(\left(\mathcal{R}_{\tilde{l}}^{\dagger}\right)_{l k}\left(\mathcal{R}_{\tilde{l}}\right)_{k l}-2\left(\mathcal{R}_{\tilde{l}}^{\dagger}\right)_{l(k+3)}\left(\mathcal{R}_{\tilde{l}}\right)_{(k+3) l}\right) \\
& \left.+\Theta(4-i) \frac{1}{6} \tan ^{2} \theta_{W} \sum_{k=4}^{6}\left(\mathcal{R}_{\tilde{l}}^{\dagger}\right)_{l k}\left(\mathcal{R}_{\tilde{l}}\right)_{k l}\right] \delta_{i j} \\
& \Gamma_{\tilde{u}_{i}, \tilde{\chi}_{l}^{+}, d_{n}}^{L}=-g_{2}\left(V_{\mathrm{CKM}}\right)_{i n} U_{l 1}+\left(V_{\mathrm{CKM}}\right)_{n(i-3)} Y_{d,(i-3)} U_{l 2} \\
& \Gamma_{\hat{\tilde{d}}_{i}, \tilde{\chi}_{l}^{+}, d_{n}}^{R}=Y_{u, n}\left(V_{\mathrm{CKM}}\right)_{n i} V_{l 2} \\
& \Gamma_{\hat{\tilde{u}}_{j}, \tilde{\chi}_{l}^{0}, u_{n}}^{L}=-\sqrt{2} g_{2}\left(\frac{1}{2} N_{l 2}-\frac{1}{6} \tan \theta_{W} N_{l 1}\right) \delta_{i n}-Y_{u, n} N_{l 4} \delta_{i(n+3)} \\
& \Gamma_{\tilde{u}_{i}, \tilde{\chi}_{l}^{0}, u_{n}}^{R}=\sqrt{2} e_{\tilde{u}} g_{2} \tan \theta_{W} N_{l 1}^{*} \delta_{i(n+3)}-Y_{u, n} N_{l 4}^{*} \delta_{i n} \\
& \Gamma_{\hat{\tilde{u}}_{i}^{*}, \hat{\tilde{u}}_{j}, \tilde{d}_{l}^{*}, \tilde{d}_{l}}=-g_{2}^{2}\left[\left(\left(V_{\mathrm{CKM}}^{\dagger}\right)_{l i}\left(V_{\mathrm{CKM}}\right)_{j l} \Theta(4-l)-\frac{3}{4} \delta_{i j}+\frac{1}{12} \tan ^{2} \theta_{W} \delta_{i j}\right)\right. \\
& \times \Theta(4-j) \Theta(4-i) \sum_{k=1}^{3}\left(\mathcal{R}_{\tilde{d}}\right)_{l k}\left(\mathcal{R}_{\tilde{d}}^{\dagger}\right)_{k l} \\
& -\frac{1}{3} \tan ^{2} \theta_{W} \delta_{i j} \Theta(j-3) \Theta(i-3) \sum_{k=1}^{3}\left(\mathcal{R}_{\tilde{d}}\right)_{l k}\left(\mathcal{R}_{\tilde{d}}^{\dagger}\right)_{k l} \\
& +\frac{1}{6} \tan ^{2} \theta_{W} \delta_{i j} \Theta(4-j) \Theta(4-i) \sum_{k=4}^{6}\left(\mathcal{R}_{\tilde{d}}\right)_{l k}\left(\mathcal{R}_{\tilde{d}}^{\dagger}\right)_{k l} \\
& \left.-\frac{2}{3} \tan ^{2} \theta_{W} \delta_{i j} \Theta(j-3) \Theta(i-3) \sum_{k=4}^{6}\left(\mathcal{R}_{\tilde{d}}\right)_{l k}\left(\mathcal{R}_{\tilde{d}}^{\dagger}\right)_{k l}\right] \\
& -\sum_{k=1}^{3}\left(\left(\mathcal{R}_{\tilde{d}}^{\dagger}\right)_{l k} Y_{d, k}\left(\mathcal{R}_{\tilde{d}}\right)_{(k+3) l}\right) Y_{u, i} \delta_{i(j+3)}-\sum_{k=1}^{3}\left(\left(\mathcal{R}_{\tilde{d}}^{\dagger}\right)_{l k} Y_{d, k}\left(\mathcal{R}_{\tilde{d}}\right)_{(k+3) l}\right)^{*} Y_{u, i} \delta_{(i+3) j}
\end{aligned}
$$




$$
\begin{aligned}
\Gamma_{\hat{u}_{i}^{*}, \hat{u}_{j}, \tilde{u}_{l}^{*}, \tilde{u}_{l}=} & \frac{4}{3} g_{3}^{2}\left(\mathcal{R}_{\tilde{u}}\right)_{l i}\left(\mathcal{R}_{\tilde{u}}^{\dagger}\right)_{j l}[\Theta(i-3) \Theta(j-3)+\Theta(4-i) \Theta(4-j) \\
& -\Theta(i-3) \Theta(4-j)-\Theta(4-i) \Theta(j-3)] \\
& -\frac{g_{2}^{2}}{36}\left[\left[\left(9+\tan ^{2} \theta_{W}\right)\left(\mathcal{R}_{\tilde{u}}\right)_{l i}\left(\mathcal{R}_{\tilde{u}}^{\dagger}\right)_{j l}+3 \sum_{k=1}^{3}\left(\left(9+\tan ^{2} \theta_{W}\right)\left(\mathcal{R}_{\tilde{u}}\right)_{l k}\left(\mathcal{R}_{\tilde{u}}^{\dagger}\right)_{k l}\right.\right.\right. \\
& \left.\left.-4 \tan ^{2} \theta_{W}\left(\mathcal{R}_{\tilde{u}}\right)_{l(k+3)}\left(\mathcal{R}_{\tilde{u}}^{\dagger}\right)_{(k+3) l}\right) \delta_{i j}\right] \Theta(4-i) \Theta(4-j) \\
& +4 \tan ^{2} \theta_{W}\left[4\left(\mathcal{R}_{\tilde{u}}\right)_{l i}\left(\mathcal{R}_{\tilde{u}}^{\dagger}\right)_{j l}\right. \\
& \left.+3 \sum_{k=1}^{3}\left(-\left(\mathcal{R}_{\tilde{u}}\right)_{l k}\left(\mathcal{R}_{\tilde{u}}^{\dagger}\right)_{k l}+4\left(\mathcal{R}_{\tilde{u}}\right)_{l(k+3)}\left(\mathcal{R}_{\tilde{u}}^{\dagger}\right)_{(k+3) l}\right) \delta_{i j}\right] \Theta(i-3) \Theta(j-3) \\
& \left.-4 \tan ^{2} \theta_{W}\left(\mathcal{R}_{\tilde{u}}\right)_{l i}\left(\mathcal{R}_{\tilde{u}}^{\dagger}\right)_{j l}(\Theta(4-i) \Theta(j-3)+\Theta(i-3) \Theta(4-j))\right] \\
& -3 Y_{u, i} \delta_{i(j-3)} \Theta(4-i) \Theta(j-3) \sum_{k=1}^{3} Y_{u, k}\left(\mathcal{R}_{\tilde{u}}\right)_{l k}\left(\mathcal{R}_{\tilde{u}}^{\dagger}\right)_{(k+3) l} \\
& -3 Y_{u, j} \delta_{(i-3) j} \Theta(4-j)_{(} \Theta(i-3) \sum_{k=1}^{3} Y_{u, k}\left(\mathcal{R}_{\tilde{u}}\right)_{l k}\left(\mathcal{R}_{\tilde{u}}^{\dagger}\right)_{(k+3) l} \\
& -Y_{u, i} Y_{u, j}\left(\mathcal{R}_{\tilde{u}}\right)_{l(i+3)}\left(\mathcal{R}_{\tilde{u}}^{\dagger}\right)_{(j+3) l} \Theta(4-j) \Theta(4-i) \\
& -Y_{u,(i-3)} Y_{u,(j-3)}\left(\mathcal{R}_{\tilde{u}}\right)_{l(i-3)}\left(\mathcal{R}_{\tilde{u}}^{\dagger}\right)_{(j-3) l} \Theta(j-3) \Theta(i-3)
\end{aligned}
$$

with

$$
\begin{aligned}
\Theta(i) & =\left\{\begin{array}{l}
1, i>0 \\
0, i \leq 0
\end{array}\right. \\
D_{\tilde{q}} & \left.=\frac{g_{2}^{2}}{4 \cos ^{2} \theta_{W}}\left[\left(T_{3}^{\tilde{q}}-e_{\tilde{q}} \sin ^{2} \theta_{W}\right) \Theta(4-i)+e_{\tilde{q}} \sin ^{2} \theta_{W} \Theta(i-3)\right)\right], \\
D_{\tilde{q}}^{\prime} & \left.=\frac{g_{2}^{2}}{4 \cos ^{2} \theta_{W}}\left[\left(T_{3}^{\tilde{q}} \cos 2 \theta_{W}+e_{\tilde{q}} \sin ^{2} \theta_{W}\right) \Theta(4-i)-e_{\tilde{q}} \sin ^{2} \theta_{W} \Theta(i-3)\right)\right], \\
h_{l}^{0} & =\left(h^{0}, H^{0}\right), \\
R^{h^{0}} & =\left(\begin{array}{cc}
-\sin \alpha \cos \alpha \\
\cos \alpha & \sin \alpha
\end{array}\right), \\
A_{l}^{0} & =\left(G^{0}, A^{0}\right), \\
R^{A^{0}} & =\left(\begin{array}{cc}
\cos \beta & \sin \beta \\
-\sin \beta \cos \beta
\end{array}\right), \\
H_{l}^{+} & =\left(G^{+}, H^{+}\right), \\
R^{H^{+}} & =R^{A^{0}}
\end{aligned}
$$


$U, V$ are the chargino mixing matrices, $N$ is the neutralino mixing matrix, $Y_{d}, Y_{u}$ and $Y_{l}$ are the usual fermion Yukawa couplings. We are summing over repeated indices and usually it is assumed that in case of $3 \times 3$ matrices the range of the summation is restricted to 3 . The one-loop scalar functions $A_{0}, B_{0}, F_{0}$ and $G_{0}$ can be found in appendix B of ref. [39], where also the tree-level masses for charginos, neutralinos, and the Higgs-bosons are given. The slepton and sneutrino masses including flavour violation can be found, e.g., in refs. [40, 41].

Open Access. This article is distributed under the terms of the Creative Commons Attribution Noncommercial License which permits any noncommercial use, distribution, and reproduction in any medium, provided the original author(s) and source are credited.

\section{References}

[1] LHC/LC Study Group collaboration, G. Weiglein et al., Physics interplay of the LHC and the ILC, Phys. Rept. 426 (2006) 47 [hep-ph/0410364] [SPIRES].

[2] G. D'Ambrosio, G.F. Giudice, G. Isidori and A. Strumia, Minimal flavour violation: An effective field theory approach, Nucl. Phys. B 645 (2002) 155 [hep-ph/0207036] [SPIRES].

[3] J.F. Donoghue, H.P. Nilles and D. Wyler, Flavor Changes in Locally Supersymmetric Theories, Phys. Lett. B 128 (1983) 55 [SPIRES].

[4] L.J. Hall, V.A. Kostelecky and S. Raby, New Flavor Violations in Supergravity Models, Nucl. Phys. B 267 (1986) 415 [SPIRES].

[5] F. Gabbiani and A. Masiero, FCNC in Generalized Supersymmetric Theories, Nucl. Phys. B 322 (1989) 235 [SPIRES].

[6] F. del Aguila et al., Collider aspects of flavour physics at high Q, Eur. Phys. J. C 57 (2008) 183 [arXiv:0801.1800] [SPIRES].

[7] J. Guasch and J. Solà, FCNC top quark decays: A door to SUSY physics in high luminosity colliders?, Nucl. Phys. B 562 (1999) 3 [hep-ph/9906268] [SPIRES].

[8] T. Hahn, W. Hollik, J.I. Illana and S. Penaranda, Interplay between $H \rightarrow b$ anti-s and $b \rightarrow s \gamma$ in the MSSM with non-minimal flavour violation, hep-ph/0512315 [SPIRES].

[9] J. Cao, G. Eilam, K.-i. Hikasa and J.M. Yang, Experimental constraints on stop-scharm flavor mixing and implications in top-quark FCNC processes, Phys. Rev. D 74 (2006) 031701 [hep-ph/0604163] [SPIRES].

[10] J.J. Cao et al., SUSY-induced FCNC top-quark processes at the Large Hadron Collider, Phys. Rev. D 75 (2007) 075021 [hep-ph/0702264] [SPIRES].

[11] G. Bozzi, B. Fuks, B. Herrmann and M. Klasen, Squark and gaugino hadroproduction and decays in non- minimal flavour violating supersymmetry, Nucl. Phys. B 787 (2007) 1 [arXiv:0704.1826] [SPIRES].

[12] B. Fuks, B. Herrmann and M. Klasen, Flavour Violation in Gauge-Mediated Supersymmetry Breaking Models: Experimental Constraints and Phenomenology at the LHC, Nucl. Phys. B 810 (2009) 266 [arXiv: 0808.1104] [SPIRES].

[13] T. Hurth and W. Porod, Non-diagonal flavour observables in B and collider physics, Eur. Phys. J. C 33 (2004) s764 [hep-ph/0311075] [SPIRES]. 
[14] T. Hurth and W. Porod, Flavour violating squark and gluino decays, JHEP 08 (2009) 087 [arXiv: 0904 .4574] [SPIRES].

[15] A. Bartl et al., Impact of squark generation mixing on the search for gluinos at LHC, Phys. Lett. B 679 (2009) 260 [arXiv:0905.0132] [SPIRES].

[16] A. Bartl, H. Eberl, B. Herrmann, K. Hidaka, W. Majerotto and W. Porod, Impact of squark generation mixing on the search for squarks decaying into fermions at $\mathrm{LHC}$, arXiv: 1007.5483 [SPIRES].

[17] M. Ciuchini, E. Franco, A. Masiero and L. Silvestrini, $b \rightarrow s$ transitions: A new frontier for indirect SUSY searches, Phys. Rev. D 67 (2003) 075016 [Erratum-ibid. D 68 (2003) 079901] [hep-ph/0212397] [SPIRES].

[18] S. Heinemeyer, W. Hollik, F. Merz and S. Penaranda, Electroweak precision observables in the MSSM with non- minimal flavor violation, Eur. Phys. J. C 37 (2004) 481 [hep-ph/0403228] [SPIRES].

[19] M. Ciuchini and L. Silvestrini, Upper bounds on SUSY contributions to $b \rightarrow s$ transitions from B/s - anti-B/s mixing, Phys. Rev. Lett. 97 (2006) 021803 [hep-ph/0603114] [SPIRES].

[20] J.S. Hagelin, S. Kelley and T. Tanaka, Supersymmetric flavor changing neutral currents: Exact amplitudes and phenomenological analysis, Nucl. Phys. B 415 (1994) 293 [SPIRES].

[21] F. Gabbiani, E. Gabrielli, A. Masiero and L. Silvestrini, A complete analysis of FCNC and CP constraints in general SUSY extensions of the standard model, Nucl. Phys. B 477 (1996) 321 [hep-ph/9604387] [SPIRES].

[22] M. Ciuchini et al., D - $\bar{D}$ mixing and new physics: General considerations and constraints on the MSSM, Phys. Lett. B 655 (2007) 162 [hep-ph/0703204] [SPIRES].

[23] W. Porod, SPheno, a program for calculating supersymmetric spectra, SUSY particle decays and SUSY particle production at $e^{+} e^{-}$colliders, Comput. Phys. Commun. 153 (2003) 275 [hep-ph/0301101] [SPIRES].

[24] M. Bruhnke and T. Hahn, A Simple Way to Distribute Mathematica Evaluations, arXiv:0902.1885 [SPIRES].

[25] S.P. Martin and M.T. Vaughn, Two loop renormalization group equations for soft supersymmetry breaking couplings, Phys. Rev. D 50 (1994) 2282 [hep-ph/9311340] [SPIRES].

[26] Heavy Flavor Averaging Group collaboration, E. Barberio et al., Averages of b-hadron and c-hadron Properties at the End of 200\%, arXiv:0808.1297 [SPIRES].

[27] T. Hurth, E. Lunghi and W. Porod, Untagged $\bar{B} \rightarrow X_{s+d} \gamma C P$ asymmetry as a probe for new physics, Nucl. Phys. B 704 (2005) 56 [hep-ph/0312260] [SPIRES].

[28] M. Misiak et al., The first estimate of $B(\bar{B} \rightarrow X / s \gamma)$ at $O\left(\alpha_{s}^{2}\right)$, Phys. Rev. Lett. 98 (2007) 022002 [hep-ph/0609232] [SPIRES].

[29] Particle Data Group collaboration, C. Amsler et al., Review of particle physics, Phys. Lett. B 667 (2008) 1 [SPIRES].

[30] P. Ball and R. Fleischer, Probing new physics through B mixing: Status, benchmarks and prospects, Eur. Phys. J. C 48 (2006) 413 [hep-ph/0604249] [SPIRES].

[31] T. Huber, T. Hurth and E. Lunghi, Logarithmically Enhanced Corrections to the Decay Rate and Forward Backward Asymmetry in $\bar{B} \rightarrow X_{s} \ell^{+} \ell^{-}$, Nucl. Phys. B 802 (2008) 40 [arXiv: 0712.3009] [SPIRES]. 
[32] B.C. Allanach, A. Djouadi, J.L. Kneur, W. Porod and P. Slavich, Precise determination of the neutral Higgs boson masses in the MSSM, JHEP 09 (2004) 044 [hep-ph/0406166] [SPIRES].

[33] J.A. Aguilar-Saavedra et al., Supersymmetry parameter analysis: SPA convention and project, Eur. Phys. J. C 46 (2006) 43 [hep-ph/0511344] [SPIRES].

[34] B.C. Allanach et al., The Snowmass points and slopes: Benchmarks for SUSY searches, Eur. Phys. J. C 25 (2002) 113 [hep-ph/0202233] [SPIRES].

[35] C.F. Kolda and S.P. Martin, Low-energy supersymmetry with D term contributions to scalar masses, Phys. Rev. D 53 (1996) 3871 [hep-ph/9503445] [SPIRES].

[36] J.A. Casas and S. Dimopoulos, Stability bounds on flavor-violating trilinear soft terms in the MSSM, Phys. Lett. B 387 (1996) 107 [hep-ph/9606237] [SPIRES].

[37] F. Staub, From Superpotential to Model Files for FeynArts and CalcHep/CompHEP, Comput. Phys. Commun. 181 (2010) 1077 [arXiv:0909.2863] [SPIRES].

[38] F. Staub, Automatic Calculation of supersymmetric Renormalization Group Equations and Self Energies, arXiv:1002.0840 [SPIRES].

[39] D.M. Pierce, J.A. Bagger, K.T. Matchev and R.-j. Zhang, Precision corrections in the minimal supersymmetric standard model, Nucl. Phys. B 491 (1997) 3 [hep-ph/9606211] [SPIRES].

[40] A. Bartl et al., Test of lepton flavour violation at LHC, Eur. Phys. J. C 46 (2006) 783 [hep-ph/0510074] [SPIRES].

[41] A. Bartl et al., Impact of slepton generation mixing on the search for sneutrinos, Phys. Lett. B 660 (2008) 228 [arXiv:0709.1157] [SPIRES]. 